\title{
Numerical-symbolic exact irreducible decomposition of cyclic-12
}

\author{
Rostam Sabeti
}

\begin{abstract}
In 1992, Göran Björck and Ralf Fröberg completely characterized the solution set of cyclic- 8 . In 2001, Jean-Charles Faugère determined the solution set of cyclic-9, by computer algebra methods and Gröbner basis computation. In this paper, a new theory in matrix analysis of rank-deficient matrices together with algorithms in numerical algebraic geometry enables us to present a symbolic-numerical algorithm to derive exactly the defining polynomials of all prime ideals of positive dimension in primary decomposition of cyclic-12. Empirical evidence together with rigorous proof establishes the fact that the positive-dimensional solution variety of cyclic-12 just consists of 72 quadrics of dimension one.
\end{abstract}

\section{Introduction}

For $n \geqslant 3$, cyclic- $n$ is a series of benchmark notorious polynomial systems [7]. Let $x=$ $\left(x_{1}, \ldots, x_{n}\right) \in \mathbb{C}^{n}$. The general form of the cyclic- $n$ polynomial system is $f_{1}=0, \ldots, f_{n-1}=0$, $f_{n}=n$, where, for $1 \leqslant i \leqslant n$,

$$
f_{i}=\sum_{j=1}^{n} \prod_{k=j}^{j+i-1} x_{k},
$$

and we also identify $x_{n+1}=x_{1}, x_{n+2}=x_{2}, \ldots$

Historically, in [8], Björck established a relationship between specific solutions of (1) and a type of function which has a constant modulus and whose Fourier transform also has a constant modulus. We temporarily shift the index $j$ in (1) to start at 0 and end at $n-1$. A solution $x \in \mathbb{C}^{n}$ of (1) is called unimodular if $\left|x_{j}\right|=1$ for all $j$. Consider $z=\left(z_{0}, \ldots, z_{n-1}\right) \in$ $\mathbb{C}^{n}$ as a complex-valued function on $\mathbb{Z}_{n}:=\{0, \ldots, n-1\}$ and its Fourier transform by $\hat{z}=$ $\left(\hat{z}_{0}, \ldots, \hat{z}_{n-1}\right)$ and $\hat{z}_{\nu}=\sum_{j=0}^{n-1} z_{j} \alpha^{j \nu}$, where $\alpha=\exp (2 \pi i / n)$. If $z \neq 0$ and $\left|z_{j}\right|$ is independent of $j$, then $z$ is called equimodular. If $\hat{z}$ is also equimodular, then we say that $z$ is bi-equimodular, see [8]. For $z \in(\mathbb{C}-\{0\})^{n}$ and $x \in \mathbb{C}^{n}$, where $x_{j}=z_{j+1} / z_{j}$, Björck proved that $z$ is biequimodular if and only if $x$ is a unimodular solution of (1). For $n=4$, cyclic-4 consists of the following set of polynomials:

$$
\begin{aligned}
& p_{1}:=x_{1}+x_{2}+x_{3}+x_{4}=0, \\
& p_{2}:=x_{1} x_{2}+x_{2} x_{3}+x_{3} x_{4}+x_{4} x_{1}=0, \\
& p_{3}:=x_{1} x_{2} x_{3}+x_{2} x_{3} x_{4}+x_{3} x_{4} x_{1}+x_{4} x_{1} x_{2}=0, \\
& p_{4}:=x_{1} x_{2} x_{3} x_{4}-1=0,
\end{aligned}
$$

where $\left(x_{1}, x_{2}, x_{3}, x_{4}\right) \in \mathbb{C}^{4}$. It is easy to see that the solution variety of (2) consists of two curves

$$
V_{1}:=\left\{\left(x_{1}, x_{2}, x_{3}, x_{4}\right) \in \mathbb{C}^{4}: x_{1}+x_{3}=0, x_{2}+x_{4}=0, x_{1} x_{2}-1=0\right\}
$$

and

$$
V_{2}:=\left\{\left(x_{1}, x_{2}, x_{3}, x_{4}\right) \in \mathbb{C}^{4}: x_{1}+x_{3}=0, x_{2}+x_{4}=0, x_{1} x_{2}+1=0\right\}
$$

of dimension one. 
Nearly a decade since the advent of computer algebra software, Björck, Fröberg and Backelin partially used this software to find concise lists of solutions of cyclic- $n$ for $n \leqslant 7[\mathbf{1}, \mathbf{9}]$.

In [10], Björck and Fröberg characterized the solution set of cyclic-8 which consists of 16 components, eight of which are of degree 16 and eight of degree two plus 1152 isolated roots. In 2001, Faugère [12] determined the solution set of cyclic-9, which has six components of dimension two and degree three plus 6642 isolated roots.

For $n=12$, the system is

$$
\begin{aligned}
f_{1} & :=x_{1}+x_{2}+\ldots+x_{11}+x_{12}=0 \\
f_{2} & :=x_{1} x_{2}+x_{2} x_{3}+\ldots+x_{11} x_{12}+x_{12} x_{1}=0 \\
& \vdots \\
f_{11} & :=x_{1} x_{2} x_{3} \ldots x_{11}+x_{2} x_{3} x_{4} \ldots x_{12}+\ldots+x_{12} x_{1} x_{2} \ldots x_{10}=0 \\
f_{12} & :=x_{1} x_{2} x_{3} \ldots x_{12}-1=0 .
\end{aligned}
$$

The main goal of this paper is to derive the exact form of all defining polynomials of prime ideals of positive dimension in primary decomposition of cyclic- 12 .

Consider a polynomial system

$$
\mathbf{P}(x):=\left(p_{1}(x), \ldots, p_{n}(x)\right):=0, \quad x=\left(x_{1}, \ldots, x_{n}\right) \in \mathbb{C}^{n},
$$

where $p_{i} \in \mathbb{C}\left[x_{1}, \ldots, x_{n}\right], i=1, \ldots, n$. It is well known that, for example, see [11], the affine algebraic variety $\mathbf{V}$ of the solution set of (4), which is defined by

$$
\mathbf{V}=\mathbf{V}(\mathbf{P})=\mathbf{P}^{-1}(0)=\left\{\left(x_{1}, \ldots, x_{n}\right) \in \mathbb{C}^{n} \mid \mathbf{P}\left(x_{1}, \ldots, x_{n}\right)=0\right\},
$$

has a unique decomposition $\mathbf{V}=V_{0} \cup \ldots \cup V_{m}$ into algebraic varieties $V_{i}, V_{i} \not \subset V_{j}, i \neq j$, $0 \leqslant m \leqslant n-1$ is a finite number and $\operatorname{dim} V_{i}=i, i=0, \ldots, m$. Note that for $i=0, \ldots, m$, $V_{i}$ is the union of $i$-dimensional components $V_{i}=\bigcup_{j \in I_{i}} V_{i j}$, such that each $I_{i}$ is finite and, for $j \in I_{i}, V_{i j}$ is not contained in a union of a collection of $V_{r s}$ unless $V_{i j}$ appears in that collection. Therefore, we have

$$
\mathbf{V}=\mathbf{V}(\mathbf{P})=\bigcup_{i=0}^{m} V_{i}=\bigcup_{i=0}^{m} \bigcup_{j \in I_{i}} V_{i j}, \quad 0 \leqslant m \leqslant n-1 .
$$

In order to obtain a numerical version of this decomposition, an approach called numerical algebraic geometry pioneered by Sommese et al. $[\mathbf{1 7}, \mathbf{1 8}, \mathbf{2 5}]$ has been introduced. Among the concepts, we may consider cascade of homotopies, witness point set and numerical irreducible decomposition.

The approach is basically an embedding of the system into a larger polynomial system such that the numerical solutions of a cascade of homotopies gives us a set of generic (witness) points on positive-dimensional components of (5).

Generally, in this article we present an algorithm which takes (4) as input and (in numerical phase) implements algorithms in numerical algebraic geometry to calculate generic points on $\mathbf{V}$ and (in symbolic phase) introduces a new theory in matrix analysis that establishes a link to numerical phase. This link (Theorem 2.4), which appears in step 2 of Algorithm 1 and step 5 of the main algorithm (Algorithm 2), generates defining polynomials of the prime ideals of positive dimension in primary decomposition of $\mathbf{I}(\mathbf{V})$ as output.

The main work in numerical algebraic geometry appeared in [25], where slicing the components with generic linear subspaces helps us to get generic points of the original system as isolated solutions of another system. The divergence of many paths in this approach led people $[\mathbf{1 7}]$ to build a theoretical framework (which basically relies on variants of Bertini's theorem, see $[\mathbf{1 3}, \mathbf{2 6}])$ to get a less-expensive numerical scheme. For the first time, interpolating polynomials have been used to sample a component (see $[\mathbf{1 8}, \mathbf{2 1}])$. 
Numerical intersection of two components of two separate systems or two components of a single system has been done via diagonal homotopies in $[\mathbf{2 2}, \mathbf{2 4}]$. Further aspects of numerical irreducible decomposition could be found in [19].

For $0 \leqslant i \leqslant n$ and $x \in \mathbb{C}^{n}, \mathbf{L}_{i}(x):=\left\{L_{1}(x), \ldots, L_{i}(x)\right\}$ stands for a set of $i$ linear hyperplanes $L_{1}, \ldots, L_{i}$ in $\mathbb{C}^{n}$ with random coefficients. It is known that the overdetermined system $\left\{\mathbf{P}(x)=0, \mathbf{L}_{i}(x)=0\right\}$ has isolated solutions if and only if the solutions lie on a component $V_{i}$ of $\mathbf{V}$ of dimension $i$, and the number of intersection points is $\operatorname{deg}\left(V_{i}\right)$ (see [26]). We denote this set by $\widehat{W}_{i}$; it is called the witness point superset for dimension $i$. Generally, for $1 \leqslant i \leqslant n-2$, points on $\widehat{W}_{i}$ may lie on $\bigcup_{k>i} V_{k}$. In this case, we have $\widehat{W}_{i}=W_{i} \cup J_{i}$, where $J_{i} \cap V_{i}=\emptyset$. We call $W_{i}$ and $J_{i}$ true witness point sets and junk points for dimension $i$, respectively. Note that $J_{n-1}=\emptyset$. Unlike the developed procedures in [26], to clear up junk points from the witness point superset we apply the heuristic idea (Kuo-Li) in [14]. In this paper, a complete revision of the Kuo-Li idea will be addressed as JunkRemove, in which we basically work on embedded systems. In terms of functionality, JunkRemove and the idea in [14] are totally different. The main advantage of JunkRemove in comparison to the junk-point removal algorithm in [26] is to avoid the construction of expensive interpolating polynomials especially for high-degree components. We denote the general form of the numerical irreducible decomposition of (5) by

$$
\mathbf{W}=\bigcup_{i=0}^{m} \bigcup_{j \in I_{i}} W_{i j} .
$$

\section{Main algorithm}

\subsection{Numerical irreducible decomposition}

Fix $1 \leqslant i \leqslant n-1$. For $x=\left(x_{1}, \ldots, x_{n}\right) \in \mathbb{C}^{n}, z=\left(z_{1}, \ldots, z_{i}\right) \in \mathbb{C}^{i}$, define $\xi_{i}:=(x, z)$ and $\xi_{0}:=x$. As in $[\mathbf{1 7}]$, we consider

$$
\mathcal{P}_{i}\left(\xi_{i}\right):=\left\{\begin{array}{l}
p_{1}(x)+\lambda_{1,1} z_{1}+\ldots+\lambda_{1, i} z_{i}:=0 \\
\vdots \\
p_{n}(x)+\lambda_{n, 1} z_{1}+\ldots+\lambda_{n, i} z_{i}:=0 \\
L_{1}(x)+z_{1}:=0 \\
\vdots \\
L_{i}(x)+z_{i}:=0
\end{array}\right.
$$

where, for $1 \leqslant j \leqslant i, \lambda_{i j}$ are random complex numbers, and we call it an embedded system.

For $0 \leqslant t \leqslant 1$, a series of homotopies joining embedded systems defined by

$$
H_{i}\left(\xi_{i}, t\right)=(1-t) \mathcal{P}_{i}\left(\xi_{i}\right)+t\left(\begin{array}{c}
\mathcal{P}_{i-1}\left(\xi_{i-1}\right) \\
z_{i}
\end{array}\right)=0
$$

is called a cascade of homotopies. The starting level of the cascade is an arbitrary integer $i$, $1 \leqslant i=l \leqslant n-1$, in (9). For future reference in this paper, we use Cascade as an abbreviation of [17, Algorithm 1]. Also, SVD stands for a complex version of an algorithm to calculate singular pairs of various matrices, see [14].

As a brief discussion about JunkRemove, suppose that we want to remove junk points in $\widehat{W}_{j_{0}}$. We select $w_{0} \in \widehat{W}_{j_{0}}$, and then SVD calculates $k_{0}$ singular values and their associated singular vectors. In case $k_{0}=0, w_{0}$ is a true witness point. If $k_{0}=1$, then we assume $\left(\mathbf{A S}_{\mathbf{1}}\right)$ $\operatorname{dim}\left(\mathbf{X}_{w_{0}}\right)=1$, where $\mathbf{X}_{w_{0}}$ denotes the piece of component of $\mathbf{V}\left(\mathcal{P}_{j_{0}}\right)$ that contains $w_{0} . \mathbf{A} \mathbf{S}_{\mathbf{1}}$ is equivalent to stating that the solution set of $\mathcal{P}_{j_{0}}(\xi)=0$ contains a curve. To check the validity of $\mathbf{A S}_{\mathbf{1}}$, we follow this curve by a predictor-corrector scheme to a considerable length. If $k_{0}>1$, then we may set up several assumptions $\left(\mathbf{A S}_{\mathbf{k}}\right) \operatorname{dim}\left(\mathbf{X}_{w_{0}}\right)=k, k=k_{0}, \ldots, 2$, and we test them by applying an appropriate predictor-corrector scheme on augmented overdetermined systems 
built on $\mathcal{P}_{j_{0}}(\xi)=0$. Then when we add $k$ new generic hyperplanes, $\mathbf{a}_{j}\left(\xi-w_{0}\right)=0, j=1, \ldots, k$, where $\mathbf{a}_{1}, \ldots, \mathbf{a}_{k}$ are random complex numbers. In this revised version, the extensive body of the JunkRemove algorithm is eliminated.

The following example explains JunkRemove in detail.

Example 1 (Simulated example for JunkRemove). Assume $n=10, l=8$. The numerical irreducible decomposition (NID) of this system is given by

$$
\widehat{W}=\left(\bigcup_{i=0}^{7} \widehat{W}_{i}\right) \cup W_{8} .
$$

We denote the exact irreducible decomposition by $\mathbf{V}=\bigcup_{i=0}^{8} V_{i}, \operatorname{dim}\left(V_{i}\right)=i$.

Suppose that we are in the middle of the junk-point removal process, and we have already removed junk points from $\widehat{W}_{6}$ and $\widehat{W}_{7}$. So, we have identified true witness point sets $W_{6}$ and $W_{7}$. This means that our current NID is formed as $\widehat{W}=\left(\bigcup_{i=0}^{5} \widehat{W}_{i}\right) \cup W_{6} \cup W_{7} \cup W_{8}$.

We pick $w_{0} \in \widehat{W}_{5}$. We want to determine whether $w_{0} \in J_{5}$ or not. We know that $w_{0}$ is a numerical solution of $\mathcal{P}_{i}(\xi)=0$ in (8) for $i=5$.

Suppose that $w_{0}$ is an approximation of the generic point $\bar{w}_{0}=\left(\bar{x}_{0}, 0\right)$ on $\mathbf{V}$, where $\bar{x}_{0} \in \mathbb{C}^{10}$. To form this example, we let $\bar{x}_{0} \in V_{7}$ denote the original location of $\bar{x}_{0}$, and $\overline{\mathbf{X}}_{\bar{x}_{0}} \subset V_{7}$ be the piece of exact irreducible component on which $\bar{x}_{0}$ lies. Also, denote the overdetermined system $\left\{p_{1}\left(\bar{x}_{0}\right)=\ldots=p_{10}\left(\bar{x}_{0}\right)=L_{1}\left(\bar{x}_{0}\right)=\ldots=L_{5}\left(\bar{x}_{0}\right)=0\right\}$ by $\mathbf{P}_{5}\left(\bar{x}_{0}\right)=0$. Denote the number of (exactly) zero singular values of $J \mathbf{P}_{5}\left(\bar{x}_{0}\right)$ by $K_{0}$. Clearly, $K_{0}=2$.

Note that we have numerical values like $w_{0}:=\left(x_{0}, z_{1}, \ldots, z_{5}\right)$, where $x_{0} \in \mathbb{C}^{10}$ and $\left|z_{i}\right| \approx$ $10^{-16}, i=1, \ldots, 5$.

We use SVD to compute all $k_{0}$ singular pairs $\left(\sigma_{1}, \mathbf{v}_{1}\right), \ldots,\left(\sigma_{k_{0}}, \mathbf{v}_{k_{0}}\right)$ of $J \mathcal{P}_{5}\left(w_{0}\right)$, where $\sigma_{1} \leqslant \ldots \leqslant \sigma_{k_{0}}$ are less than a preassigned small threshold $\bar{\sigma}>0$. Since we deal with numerical calculations, we may easily have $k_{0}>K_{0}$ or $k_{0} \leqslant K_{0}$. Usually, we should take a moderately small threshold.

Let $\mathbf{X}_{w_{0}}$ be a piece of numerical irreducible component of $\mathbf{V}\left(\mathcal{P}_{5}\right)$ that contains $w_{0}$. In JunkRemove, we approximate $\operatorname{dim}\left(\overline{\mathbf{X}}_{\bar{x}_{0}}\right)$ by $\operatorname{dim}\left(\mathbf{X}_{w_{0}}\right)$.

Since $k_{0}$ may not exactly determine $\operatorname{dim}\left(\mathbf{X}_{w_{0}}\right)$, we rely on other criteria to calculate $\operatorname{dim}\left(\mathbf{X}_{w_{0}}\right)$ more accurately. These criteria consist of testing several assumptions. Assume $k_{0}=3>K_{0}=2$.

Suppose that $\sigma_{1}$ and $\sigma_{2}$ are very close to zero, and the singular pair $\left(\sigma_{3}, \mathbf{v}_{3}\right)$ is a poor approximation. At the beginning, we made the assumption $\mathbf{A S}_{3}: \operatorname{dim}\left(\mathbf{X}_{w_{0}}\right)=3$.

Having assumed $\mathbf{A S}_{3}$ means that another generic linear hyperplane of codimension two in $\mathbb{C}^{15}$, which passes through $w_{0}$ like

$$
\left\{\begin{array}{l}
\mathbf{a}_{1}^{H}\left(\xi-w_{0}\right)=0 \\
\mathbf{a}_{2}^{H}\left(\xi-w_{0}\right)=0
\end{array}\right.
$$

with $\mathbf{a}_{1}, \mathbf{a}_{2} \in \mathbb{C}^{15}$ random, intersects $\mathcal{P}_{5}(\xi)=0$ at a one-dimensional solution set $\mathbf{Y}_{w_{0}}$ of

$$
\overline{\mathcal{P}}_{5}(\xi):=\left\{\begin{array}{l}
\mathcal{P}_{5}(\xi)=0, \\
\mathbf{a}_{1}^{H}\left(\xi-w_{0}\right)=0 \\
\mathbf{a}_{2}^{H}\left(\xi-w_{0}\right)=0 .
\end{array}\right.
$$

To test $\mathbf{A S}_{3}$, we need to follow a curve $\{x(t)\}$ in $\mathbf{Y}_{w_{0}} \subset \mathbf{V}\left(\overline{\mathcal{P}}_{5}\right)$ by a predictor-corrector scheme. Since $\overline{\mathcal{P}}_{5}(\xi)$ vanishes along the curve, the tangent to the curve is in the kernel of $J \overline{\mathcal{P}}_{5}(x(t))$, $t \in \mathbb{R}$. We know that $\operatorname{rank}\left(J \mathcal{P}_{5}(x(t))\right)=12$ for all $t \in \mathbb{R}$. Then

$$
\operatorname{rank}\left(J \overline{\mathcal{P}}_{5}(x(t))\right):=\operatorname{rank}\left(\left[\begin{array}{c}
J \mathcal{P}_{5}(x(t)) \\
\mathbf{a}_{1}^{H} \\
\mathbf{a}_{2}^{H}
\end{array}\right]\right)=14 .
$$


Evidently, there is a unit vector $\mathbf{u}$ in $\mathbb{C}^{15}$ with $J \overline{\mathcal{P}}_{5}\left(w_{0}\right) \mathbf{u}=0$. Obviously, there are complex numbers $\alpha_{1}, \alpha_{2}$ and $\alpha_{3}$ such that $\mathbf{u}:=\alpha_{1} \mathbf{v}_{1}+\alpha_{2} \mathbf{v}_{2}+\alpha_{3} \mathbf{v}_{3}$. The term $\alpha_{3} \mathbf{v}_{3}$ is a problematic term, because the pair $\left(\sigma_{3}, \mathbf{v}_{3}\right)$ is a poor approximation. We predict the next point in $\mathbf{Y}_{w_{0}}$ as $\bar{\xi}=w_{0}+\delta \mathbf{u}$, for an initial step size $\delta=\delta_{0}>0$. To solve $\widetilde{\mathcal{P}}_{j_{0}}(\xi)=0$, this predicted point serves as the initial point to the following Gauss-Newton correction iteration:

$$
\xi^{0}=\bar{\xi} ; \quad \xi^{m+1}=\xi^{m}-\left[J \widetilde{\mathcal{P}}_{5}\left(\xi^{m}\right)\right]^{\dagger} \widetilde{\mathcal{P}}_{5}\left(\xi^{m}\right), \quad m=0,1, \ldots,
$$

where

$$
\widetilde{\mathcal{P}}_{5}(\xi)=\left\{\begin{array}{l}
\overline{\mathcal{P}}_{5}(\xi), \\
\mathbf{u}^{H}\left(\xi-w_{0}\right) .
\end{array}\right.
$$

Most of the time, poor approximations of singular pairs like $\left(\sigma_{3}, \mathbf{v}_{3}\right)$, cause non-convergence of (10). In this case, we reject $\mathbf{A S}_{3}$ and we assume $\mathbf{A S}_{2}: \operatorname{dim}\left(\mathbf{X}_{w_{0}}\right)=2$. Set

$$
\overline{\mathcal{P}}_{5}(\xi):=\left\{\begin{array}{l}
\mathcal{P}_{5}(\xi)=0, \\
\mathbf{a}_{1}^{H}\left(\xi-w_{0}\right)=0 .
\end{array}\right.
$$

Again we calculate singular pairs of $J \overline{\mathcal{P}}_{5}\left(w_{0}\right)$. This time, since we introduced a generic hyperplane, experience showed that the chance of having miscalculation of singular pairs diminished by a considerable amount. $K_{0}=2 \operatorname{implies} \operatorname{rank}\left(J \overline{\mathcal{P}}_{5}\left(w_{0}\right)\right)=1$, and the only singular vector of $\overline{\mathcal{P}}_{5}\left(w_{0}\right)$ will be taken as $\mathbf{u}$. Then we start the predictor-corrector scheme by letting $\bar{\xi}=w_{0}+\delta \mathbf{u}$ for an initial step size $\delta=\delta_{0}>0$.

For a preassigned tolerance $\epsilon>0$, if the correction (10) converges to $\xi_{1}^{*}$ with $\left\|\mathcal{P}_{5}\left(\xi_{1}^{*}\right)\right\|_{2}<\epsilon$, then $\xi_{1}^{*}$ will be taken as a numerical solution of $\mathcal{P}_{5}(\xi)=0$, which in turn implies $\overline{\mathcal{P}}_{5}\left(\xi_{1}^{*}\right)=0$. In the case of non-convergence, we cut the step size by half and repeat the above procedure until convergence. If $\delta$ is less than some small $\bar{\delta}$, then we claim final non-convergence.

The above prediction-correction procedure will be repeated at $\xi_{1}^{*}$ (which means that the iteration function (11) will be changed via replacement of $w_{0}$ by $\left.\xi_{1}^{*}\right)$. Since the rank of $J \overline{\mathcal{P}}_{5}\left(\xi_{1}^{*}\right)$ is 14 , it must have a very small singular value. We calculate the unit singular vector $\mathbf{u}$ of $J \overline{\mathcal{P}}_{5}\left(\xi_{1}^{*}\right)$ corresponding to its smallest singular value that is less than $\bar{\sigma}>0$. The sign of $\mathbf{u}$ must be consistent with $\xi_{1}^{*}-\xi_{0}^{*}$, which makes the tangent appropriate as our next prediction step at $\xi_{1}^{*}$. We continue to produce consecutively a sequence of points $\xi_{1}^{*}, \xi_{2}^{*}, \ldots$, along with successful step sizes $\delta_{1}, \delta_{2}, \ldots$ The assumption $\mathbf{A S}_{2}$ will be accepted if the accumulated successful step sizes reach a sufficient length. From the new iteration function (11), it is clear that (see above explanation)

$$
\left\|\xi_{i}^{*}-\xi_{i-1}^{*}\right\|_{2} \geqslant \delta_{i}, \quad i=1,2, \ldots
$$

Therefore,

$$
\sum_{i=1}^{m}\left\|\xi_{i}^{*}-\xi_{i-1}^{*}\right\|_{2} \geqslant \sum_{i=1}^{m} \delta_{i} \equiv S(m), \quad m>1 .
$$

We admit the existence of $\mathbf{X}_{w_{0}}$ (which implies that $w_{0}$ is a junk point or $w_{0} \in J_{5}$ ) if $S(m)>\eta$, where $\eta>0$ is a preassigned length for a certain $m>1$.

Recent advances (see [2]) in this field shed light on the challenging problem of junk-point removal for polynomial systems of moderate size.

REMARK 1. The point of departure in [2] is $\mathbf{p}$ a numerical approximation of a point on $V(P)$. As the output of Algorithm 3, page 3617 in [2] shows, it seems that it returns the dimension and component number of an irreducible component of which $\mathbf{p}$ is a member. Rich theoretical setting makes this algorithm valuable for polynomial systems of moderate size. In our large-scale problem, cyclic-12, adding one more variable (for homogenization) makes the 
original problem of calculating witness points for a system of size $n=13$ into an impossible or at least extremely time-consuming problem. In order to apply this method for our case, another complication is the construction of multiplicity matrices which deal with partial derivatives for a set of 13 variables. The heuristic nature of the Kuo-Li idea in the junk-point removal process, compared to the local dimension test algorithm, appears in [2, Example 3.2, p. 3619].

Let the range of $i$ and $j$ be as in (7). In order to break up each witness point superset $\widetilde{W}_{i}$ into a union of witness points on numerical irreducible components $W_{i j}$ in (7), we apply monodromy grouping with linear trace validation $[\mathbf{2 0}, \mathbf{2 6}]$. In our implementation of monodromy and linear trace, we solve the necessary overdetermined systems which consist of a polynomial system $\mathbf{P}(x)=0$ together with a set of generic hyperplanes, by the following form of so-called overdetermined homotopy

$$
\mathbf{H}(x, t)=(1-t)\left(\begin{array}{c}
\mathbf{P}(x) \\
\mathbf{L}_{1}(x)
\end{array}\right)+t\left(\begin{array}{c}
\mathbf{P}(x) \\
\mathbf{L}_{2}(x)
\end{array}\right)=0 .
$$

Instead of matrix inversion in Newton correction as in regular continuation, we apply pseudoinversion of matrices and Gauss-Newton iteration as corrector. In this homotopy, $\mathbf{L}_{1}$ is the original set of linear generic hyperplanes used in (8), and $\mathbf{L}_{2}$ is another arbitrary set. So, every time, we start at solutions of $\left\{\mathbf{P}=0, \mathbf{L}_{1}=0\right\}$ (that is, the current set of numbered true witness points) and we reach solutions of $\left\{\mathbf{P}=0, \mathbf{L}_{2}=0\right\}$ and then move backward by changing the systems in (12) as $\left\{\mathbf{P}=0, \mathbf{L}_{1}=0\right\} \rightarrow\left\{\mathbf{P}=0, \mathbf{L}_{2}=0\right\}$ and $\left\{\mathbf{P}=0, \mathbf{L}_{2}=0\right\} \rightarrow \alpha\{\mathbf{P}=0$, $\left.\mathbf{L}_{1}=0\right\}$, where $\alpha$ is a random complex number. Our implementation of monodromy and trace, together with JunkRemove, makes our algorithm on numerical irreducible decomposition.

\subsection{Deficiency pattern for rank-deficient matrices}

This section introduces new facts in matrix theory. This theory provides the main aim of this paper, which is a link between numerical phase to symbolic phase of the main algorithm. The fundamental fact in Theorem 2.4 establishes this link. Throughout this section, we fix integers $m, n, k$ with $0<m \leqslant n$ and $k>0$. Suppose $\mathbf{A}=\left[\mathbf{A}_{1}, \ldots, \mathbf{A}_{n}\right] \in \mathbb{C}^{m \times n}$ with $\operatorname{rank}(\mathbf{A})=k$ and $\operatorname{NullSpace}(\mathbf{A})=\operatorname{span}\left\{\mathbf{v}_{1}, \ldots, \mathbf{v}_{n-k}\right\}$, where $\mathbf{v}_{j}=\left(v_{j 1}, \ldots, v_{j n}\right)^{T} \in \mathbb{C}^{n}$, for $j=1, \ldots, n-k$, are called zero vectors (of $\mathbf{A}$ ). The proof of the following lemma is simple. We state it for convenience.

Lemma 2.1. With the above notation, suppose $\Omega:=\left\{1 \leqslant i \leqslant n: v_{j i}=0\right.$ for all $j=$ $1, \ldots, n-k\}$. Then $\mathcal{I}(\mathbf{A}):=\left\{\mathbf{A}_{i}: i \in \Omega\right\}$ is a linearly independent subset of $\mathbb{C}^{m}$.

Proof. $\quad$ Let $l>0$ and $\Omega=\left\{r_{1}<\ldots<r_{l}\right\}$. For $j=1, \ldots, l$, let $\alpha_{r_{j}} \in \mathbb{F}$ be such that $\alpha_{r_{1}} \mathbf{A}_{r_{1}}+$ $\alpha_{r_{2}} \mathbf{A}_{r_{2}}+\ldots+\alpha_{r_{l}} \mathbf{A}_{r_{l}}=0$. Therefore,

$$
\overline{\mathbf{v}}=\left(0, \ldots, 0, \alpha_{r_{1}}, 0, \ldots, 0, \alpha_{r_{2}}, 0, \ldots, 0, \alpha_{r_{l}}, 0, \ldots, 0\right)^{T}
$$

is in NullSpace(A), where $\alpha_{r_{j}}$ is in the $r_{j}$ th position for $j=1, \ldots, l$. Therefore, $\overline{\mathbf{v}}$ is a linear combination of $\mathbf{v}_{1}, \ldots, \mathbf{v}_{n-k}$. Since $v_{j r_{t}}=0$ for $j=1, \ldots, n-k$ and $t=1, \ldots, l$, then $\alpha_{r_{j}}=0$, $j=1, \ldots, l$. The same reason shows that all $\mathbf{A}_{r_{1}}, \ldots, \mathbf{A}_{r_{l}}$ are non-zero.

The indices in $\Omega$ can be identified by just comparing any set of zero vectors. We define the elements in $\mathcal{I}(\mathbf{A})$ as isolated columns of $\mathbf{A}$. The other columns of $\mathbf{A}$ are called non-isolated.

Any arbitrary linear combination of columns of $\mathbf{A}$ corresponds to a zero vector none of whose non-zero elements corresponds to an isolated column of A. See the proof of the next lemma.

Lemma 2.2. With the above notation, $\# \mathcal{I}(\mathbf{A})<k$. 
Proof. Let $\# \mathcal{I}(\mathbf{A})=k$. Since $\operatorname{rank}(\mathbf{A})=k$, Lemma 2.1 shows that for non-zero $\mathbf{A}_{l} \notin \mathcal{I}(\mathbf{A})$, $\mathcal{I}(\mathbf{A}) \cup\left\{\mathbf{A}_{l}\right\}$ is a linearly dependent set. So, there exists a zero vector $\mathbf{w}$ such that $w_{l} \neq 0$ and $w_{i_{0}} \neq 0$, where $\mathbf{A}_{i_{0}}$ is an isolated column. Since $w_{i_{0}} \neq 0$, Lemma 2.1 gives a contradiction.

Theorem 2.3. With the above notation, let $\mathbf{N}:=\mathbf{N}(\mathbf{A}):=\left[\mathbf{v}_{1}, \ldots, \mathbf{v}_{n-k}\right] \in \mathbb{C}^{n \times(n-k)}$. There exists a non-singular transformation $\mathbf{P} \in \mathbb{C}^{(n-k) \times(n-k)}$ such that

$$
\mathbf{P}^{T} \mathbf{N}^{T}=\widetilde{\mathbf{N}}(\mathbf{A})=\left[\begin{array}{rrrrrrrrr} 
& 1 & & 0 & & 0 & \ldots & 0 & \\
& 0 & & 1 & & 0 & \ldots & 0 & \\
\mathbf{V}^{(0)} & 0 & \mathbf{V}^{(1)} & 0 & \mathbf{V}^{(2)} & 1 & \ldots & 0 & \mathbf{V}^{(n-k)} \\
& \vdots & & \vdots & & \vdots & \ddots & \vdots & \\
& 0 & & 0 & & 0 & & 1 &
\end{array}\right]
$$

where each 1 from left to right is in the $i_{1} t h, \ldots, i_{n-k}$ th column, respectively, for integers $i_{1}<$ $\ldots<i_{n-k}$. Moreover, for $j=0,1, \ldots, n-k$, we have $\mathbf{V}^{(j)} \in \mathbb{C}^{(n-k) \times l_{j}}$ for $l_{j} \geqslant 0\left(l_{j}=0, \mathbf{V}^{(j)}\right.$ is a degenerate block), and

$$
\mathbf{V}_{s t}^{(j)}=0, \quad s=j+1, \ldots, n-k, \quad t=1, \ldots, l_{j} .
$$

Evidently,

$$
\operatorname{rank}\left(\mathbf{N}^{T}\right)=\operatorname{rank}(\tilde{\mathbf{N}}(\mathbf{A}))=n-k
$$

Proof. Start from the upper-left corner of $\mathbf{N}^{T}$ and search for the first non-zero term in the corresponding row and apply successive Gaussian elimination to $\mathbf{N}^{T}$ from the left and, whenever it is needed, apply a row interchange permutation matrix to it. Note that column interchange is not allowed.

Equations (13) in compact matrix form are given by

$$
\mathbf{V}^{(j)}=\left[\begin{array}{c}
\mathbf{Z}^{(j)} \\
\mathbf{0}
\end{array}\right]
$$

where $\mathbf{Z}^{(j)}$ is a non-zero matrix in $\mathbb{C}^{j \times l_{j}}$.

Large-scale examples clearly show a non-trivial block structure of $\widetilde{\mathbf{N}}(\mathbf{A})$ in the above proof. See Example 3.

EXAmple 2. Suppose

$$
\mathbf{A}=\left[\begin{array}{rrrrr}
1 & -4 & 0 & -3 & 1 \\
0 & 0 & 2 & 3 & -1 \\
1 & -2 & 1 & 0 & 0 \\
-1 & -8 & -2 & -9 & 3 \\
1 & -14 & 3 & -6 & 2
\end{array}\right]
$$

We see that $\operatorname{rank}(\mathbf{A})=3$ and $\mathbf{v}_{1}=(0,1,2,0,4)^{T}$ and $\mathbf{v}_{2}=(0,0,0,1,3)^{T}$ are two basis vectors in Nullspace $(\mathbf{A})$. Clearly, the representation of Theorem 2.3 in this example reflects on columns of $\mathbf{A}$ as $\mathbf{A}_{2}+2 \mathbf{A}_{3}+4 \mathbf{A}_{5}=0$ and $\mathbf{A}_{4}+3 \mathbf{A}_{5}=0$, and also we have

$$
\tilde{\mathbf{N}}(\mathbf{A})=\left[\begin{array}{lllll}
0 & 1 & 2 & 0 & 4 \\
0 & 0 & 0 & 1 & 3
\end{array}\right]
$$

$\mathcal{I}(\mathbf{A})=\left\{\mathbf{A}_{1}\right\}$, which means that the only isolated column of $\mathbf{A}$ is $\mathbf{A}_{1}$ and $\Omega=\{1\}$. The other parameters of Theorem 2.3 follow as $i_{1}=2, i_{2}=4, l_{0}=1, l_{1}=1, l_{2}=1$ and also

$$
\mathbf{V}^{(0)}=\left[\begin{array}{l}
0 \\
0
\end{array}\right], \quad \mathbf{V}^{(1)}=\left[\begin{array}{l}
2 \\
0
\end{array}\right] \quad \text { and } \quad \mathbf{V}^{(2)}=\left[\begin{array}{l}
4 \\
3
\end{array}\right]
$$


Definition 1. With the above notation, suppose $i_{1}<\ldots<i_{n-k}$ and let $\widetilde{\mathbf{N}}(\mathbf{A})$ be as in Theorem 2.3, and, for $1 \leqslant j \leqslant n-k$, let $\boldsymbol{\Delta}_{i_{j}}=\left\{i_{j}<t \leqslant n:(\tilde{\mathbf{N}}(\mathbf{A}))_{i_{j}, t} \neq 0\right\}$. Then, for $1 \leqslant j \leqslant n-k$, the following unique set of linear combinations:

$$
\mathbf{A}_{i_{j}}+\sum_{t \in \mathbf{\Delta}_{i_{j}}}(\tilde{\mathbf{N}}(\mathbf{A}))_{i_{j}, t} \mathbf{A}_{t}=0
$$

of columns of $\mathbf{A}$ is called the deficiency pattern of $\mathbf{A}$. The indices $i_{1}<\ldots<i_{n-k}$ are called the leading indices of the pattern.

The deficiency pattern of $\mathbf{A}$ in Example 2 is $\mathbf{A}_{2}+2 \mathbf{A}_{3}+4 \mathbf{A}_{5}=0$ and $\mathbf{A}_{4}+3 \mathbf{A}_{5}=0$.

Example 3. A large-scale example is given by

$$
\mathbf{A}=\left[\begin{array}{rrrrrrrrrr}
-1 & 0 & 1 & 0 & -2 & 0 & 1 & 1 & 0 & 0 \\
0 & -3 & -1 & 0 & 6 & 1 & -2 & -2 & 0 & 0 \\
1 & 0 & 0 & 0 & 2 & 2 & 1 & 1 & 0 & 0 \\
-5 & 2 & 2 & 1 & 0 & -1 & 3 & -1 & 0 & 0 \\
-6 & 1 & 3 & 0 & 0 & 0 & 0 & 0 & 0 & 0 \\
4 & -2 & -2 & 1 & 0 & 0 & 0 & 0 & 0 & 0 \\
1 & 0 & 0 & -2 & 0 & 1 & 1 & 1 & 0 & 0 \\
2 & 2 & 0 & 1 & 0 & 2 & 0 & 2 & 0 & 0 \\
-4 & 1 & 1 & 3 & 6 & 1 & -2 & -2 & 0 & 0 \\
-3 & -2 & 2 & 1 & 0 & 1 & 2 & 1 & 0 & 0
\end{array}\right]
$$

It is easy to verify that $\operatorname{rank}(\mathbf{A})=6$ and

$$
\widetilde{\mathbf{N}}(\mathbf{A})=\left[\begin{array}{rrrrrrrrrr}
1 & 0 & 2 & 0 & 0 & 0 & 0 & -1 & 0 & 0 \\
0 & 0 & 0 & 0 & 1 & -2 & 0 & 2 & 0 & 0 \\
0 & 0 & 0 & 0 & 0 & 0 & 0 & 0 & 1 & 0 \\
0 & 0 & 0 & 0 & 0 & 0 & 0 & 0 & 0 & 1
\end{array}\right]
$$

In this example, we have $\mathcal{I}(\mathbf{A})=\left\{\mathbf{A}_{2}, \mathbf{A}_{4}, \mathbf{A}_{7}\right\}$ and $\Omega=\{2,4,7\}, i_{1}=1, i_{2}=5, i_{3}=9, i_{4}=10$, $l_{0}=0, l_{1}=3, l_{2}=3$ and $l_{3}=l_{4}=0 . \mathbf{V}^{(0)}, \mathbf{V}^{(3)}$ and $\mathbf{V}^{(4)}$ are degenerate and

$$
\mathbf{V}^{(1)}=\left[\begin{array}{lll}
0 & 2 & 0 \\
0 & 0 & 0 \\
0 & 0 & 0 \\
0 & 0 & 0
\end{array}\right], \quad \mathbf{V}^{(2)}=\left[\begin{array}{rrr}
0 & 0 & -1 \\
-2 & 0 & 2 \\
0 & 0 & 0 \\
0 & 0 & 0
\end{array}\right] .
$$

The deficiency pattern of $\mathbf{A}$ is as follows:

$$
\left\{\begin{array}{l}
\mathbf{A}_{1}+2 \mathbf{A}_{3}-\mathbf{A}_{8}=\mathbf{0} \\
\mathbf{A}_{5}-2 \mathbf{A}_{6}+2 \mathbf{A}_{8}=\mathbf{0} \\
\mathbf{A}_{9}=\mathbf{0} \\
\mathbf{A}_{10}=\mathbf{0}
\end{array}\right.
$$

Suppose that we calculate $n+1$ witness points $X^{(1)}, \ldots, X^{(n+1)}$ on a typical irreducible variety $V \subset \mathbf{V}$ in $\mathbb{C}^{n}$ with $\operatorname{dim}(V)>0$. Then we may easily form the following numerical matrix in $\mathbb{C}^{(n+1) \times(n+1)}$ :

$$
\mathbf{A}_{\text {LinG }}:=\left[\begin{array}{llll}
X_{1}^{(1)} & \ldots & X_{n}^{(1)} & 1 \\
\vdots & \ddots & \vdots & \vdots \\
X_{1}^{(n+1)} & \ldots & X_{n}^{(n+1)} & 1
\end{array}\right]
$$


Definition 2. With the above notation, $\mathbf{A}_{L i n G}$ is called a linear generic matrix or generic matrix of order 1 associated with $V$.

Let $\operatorname{rank}\left(\mathbf{A}_{L i n G}\right)=k$. By Theorem 2.3 , there exist integers $1 \leqslant i_{1}<\ldots<i_{n+1-k} \leqslant n+1$ such that

$$
\left(\mathbf{A}_{\text {LinG }}\right)_{i_{j}}+\sum_{t \in \mathbf{\Delta}_{i_{j}}}\left(\tilde{\mathbf{N}}\left(\mathbf{A}_{\text {LinG }}\right)\right)_{i_{j}, t}\left(\mathbf{A}_{\text {LinG }}\right)_{t}=0, \quad j=1, \ldots, n+1-k
$$

is the deficiency pattern of $\mathbf{A}_{L i n G}$. So, the linear polynomials

$$
p_{j}\left(x_{1}, \ldots, x_{n}\right)=x_{i_{j}}+\sum_{t \in \boldsymbol{\Delta}_{i_{j}}}\left(\tilde{\mathbf{N}}\left(\mathbf{A}_{L i n G}\right)\right)_{i_{j}, t} x_{t}, \quad j=1, \ldots, n+1-k
$$

vanish on $V$. Thus, we have the following theorem.

TheOREm 2.4. With the above notation, $\mathbf{I}(V)$ contains the ideal generated by the linear polynomials (17).

Once we have found linear generators, we need to find out whether the ideal generated by $p_{1}, \ldots, p_{n+1-k}$ covers the whole $\mathbf{I}(V)$ or not. We realize this by finding the generators of higher total degrees. Apparently, we want to define generic matrices built on monomials of higher total degree than one. But, the size of matrices rises. Hence, we need to eliminate some of the variables.

Remark 2. Suppose that we found $m<n$ linear generators $g_{1}, \ldots, g_{m}$ of $\mathbf{I}(V)$. Evidently, there exists a finite subset of $\{1, \ldots, n\}$, say $\left\{j_{1}, \ldots, j_{s}\right\}$, such that $g_{1}, \ldots, g_{m} \in$ $\mathbb{C}\left[y_{1}, \ldots, y_{n-s}\right]\left[x_{j_{1}}, \ldots, x_{j_{s}}\right]$, where $\left\{y_{1}, \ldots, y_{n-s}\right\}=\left\{x_{1}, \ldots, x_{n}\right\}-\left\{x_{j_{1}}, \ldots, x_{j_{s}}\right\}$.

We fix a lexicographic term order $x_{j_{1}}>\ldots>x_{j_{s}}>\ldots$ on $\left\{x_{1}, \ldots, x_{n}\right\}$. We apply a standard division algorithm $[\mathbf{1 1}]$ to obtain non-zero remainders $h_{1}, \ldots, h_{k} \in \mathbb{C}\left[y_{1}, \ldots, y_{n-s}\right]$ on division of the original polynomials of the system $\left(p_{1}, \ldots, p_{n}\right)$ by $\left\{g_{1}, \ldots, g_{m}\right\}$. To get a full set of generators, we should be able to express $h_{1}, \ldots, h_{k}$ in terms of higher-order polynomials. Therefore, the next step is to define a quadratic generic matrix or generic matrix of order two, $\mathbf{A}_{Q u a G}$. To this end, consider $l_{2}:=\left(\begin{array}{c}n-s+2 \\ 2\end{array}\right)$ generic points on $V$, say $Y^{(j)}, j=1, \ldots, l_{2}$, and then define $\mathbf{A}_{Q u a G}$ as it shows after the remark. See Section 3 for the result of cyclic-12, where we have $s=10, j_{1}=3, \ldots, j_{10}=12$ and $y_{1}=x_{1}, y_{2}=x_{2}$. We just have $h_{1}$ as the remainder of $f_{12}$ by $\left\{p_{1}, \ldots, p_{10}\right\}$ with the lexicographic order as $x_{3}>x_{4}>\ldots>x_{12}>x_{1}>x_{2}$.

To follow the discussion started in Remark 2, we let

$$
\mathbf{A}_{\text {QuaG }}=\left[\begin{array}{cccccccc}
\left(Y_{1}^{(1)}\right)^{2} & Y_{1}^{(1)} Y_{2}^{(1)} & \ldots & Y_{1}^{(1)} Y_{n-s}^{(1)} & \left(Y_{2}^{(1)}\right)^{2} & Y_{2}^{(1)} Y_{3}^{(1)} & \ldots & 1 \\
\vdots & \vdots & \ddots & \vdots & \vdots & \vdots & \ddots & \vdots \\
\left(Y_{1}^{\left(l_{2}\right)}\right)^{2} & Y_{1}^{\left(l_{2}\right)} Y_{2}^{\left(l_{2}\right)} & \ldots & Y_{1}^{\left(l_{2}\right)} Y_{n-s}^{\left(l_{2}\right)} & \left(Y_{2}^{\left(l_{2}\right)}\right)^{2} & Y_{2}^{\left(l_{2}\right)} Y_{3}^{\left(l_{2}\right)} & \ldots & 1
\end{array}\right] .
$$

This means that if, for a quadratic generic matrix $\mathbf{A}_{Q u a G}$ we have $\operatorname{rank}\left(\mathbf{A}_{Q u a G}\right)=u<l_{2}$, then there are $l_{2}-u$ quadratic generators for $\mathbf{I}(V)$.

To continue, we use the same set of variables as in the quadratic case, for third-degree generators. Clearly, by the same token, we may define generic matrices of order $i$ for $i>3$. If we continue this procedure, at any stage we have a current set of generators, say $\left\{h_{1}, \ldots, h_{q}\right\}$, whose zero set is a subset of the irreducible component involved, which is $V$. Suppose that the variety of the original system $\mathbf{P}(x)=0$ is $\mathbf{V}$. Since $V \subset \mathbf{V}$, we stop the procedure if

$$
\mathbf{I}(\mathbf{V}) \subseteq \mathbf{I}(V) \subseteq \mathbf{I}\left(\left\{h_{1}, \ldots, h_{q}\right\}\right)
$$


REMARK 3. To verify the stopping criterion (18) at any stage, we apply the division algorithm we discussed in Remark 2, to get the remainder of each polynomial in the original system $\mathbf{P}(x)=0$.

The following algorithm summarizes the whole discussion as a symbolic part of our main algorithm. As input it takes a collection of generic points $C$ on a positive-dimensional irreducible variety $V$ and, as output, it gives a set of generators for $\mathbf{I}(V)$.

\section{Algorithm 1. Exact generator}

Input: A collection $C$ of generic points on an irreducible variety $V$ with $\operatorname{dim}(V)>0$.

Output: A set of generators for $G$ for which $\mathbf{I}(G):=\mathbf{I}(V)$.

Step 0: Initialize $G:=\emptyset$. Set the current set of variables as $x_{1}, \ldots, x_{n}$ and $i:=1$.

Loop: Do While $(\mathbf{I}(V)-\mathbf{I}(G) \neq \emptyset) \quad$ 'Check condition (18)'.

Step 1: Form the generic matrix of order $i$ say $A_{i}$ based on the current set of variables.

Step 2: If $A_{i}$ is rank deficient, then

Calculate the deficiency pattern of $A_{i}$, else

exit with current $G$.

End If

Step 3: Find the corresponding generators of total degree $i$.

Step 4: Update $G:=G \cup\{$ new generators $\}$.

Step 5: If $(i=1)$, then apply the type of elimination as in Remark 2 and update the current set of variables

\section{Step 6: $i:=i+1$}

\section{End for (Loop)}

Our goal is to present an algorithm that takes the polynomial system (4) as input and calculates a set of generators for each $\mathbf{I}\left(V_{i j}\right)$ as output, where $V_{i j}$ is an exact irreducible variety in exact irreducible decomposition (6). First of all, in order to deal with generic matrices (linear, quadratic or cubic, etc.), we should be able to calculate a sufficient number of generic (or sample) points on each $V_{i j}$, given a set of true (pure) witness points $W_{i j}$ on it. We take the initial set of pure witness points in overdetermined homotopy (12). Then we circulate $\mathbf{L}_{2}(x)$ and pick up a new witness point on the variety. However, if we let them be picked up without a considerable distance, then we have trouble in finding SVD of the generic matrices involved. In order to avoid miscalculation of numerical ranks of generic matrices, we need a well-separated set of sample points. By this, we mean a set of sample points $S=\left\{w=\left(w_{1}, \ldots, w_{n}\right)\right\}$ such that for two given positive real numbers $\alpha_{2}$ and $\alpha_{\infty}$, we have

$$
\forall w, \bar{w} \in S \quad\|w-\bar{w}\|_{2} \geqslant \alpha_{2}
$$

and (or)

$$
\forall w, \bar{w} \in S \quad \min _{1 \leqslant j \leqslant n}\left|w_{j}-\bar{w}_{j}\right| \geqslant \alpha_{\infty} .
$$

The sampling algorithm will be denoted by Sample. In Sample, while we generate sample points, we filter the current set of sample points based on (19) and (20). The main algorithm follows.

\section{Algorithm 2. Main algorithm}

Input: Polynomial system $\mathbf{P}(x)=0$ and $l_{0}$ : top dimension of $\mathbf{V}$.

Output: The generators for ideals $\mathbf{I}_{i j}$, where $\mathbf{I}_{i j}:=\mathbf{I}\left(V_{i j}\right)$ and $V_{i j}$ are as in (6).

Step 0: Initialize $N:=10$ and $k:=0$.

Step 1: Find the numerical irreducible decomposition $W=\bigcup_{i=0}^{l} \bigcup_{j \in I_{i}} W_{i j}$ of $\mathbf{V}$. 
Loop: For $i$ from 1 to $l_{0}$

For $j \in I_{i}$

Step 2: $k:=k+1$

Step 3: Choose appropriate $\alpha_{2}, \alpha_{\infty}$

Step 4: Call Sample to get enough well-separated generic points on $V_{i j}$ and call the resulting set of points as $C_{i j}$

Step 5: Apply Algorithm 1 on $C_{i j}$ and get generators of $\mathbf{I}_{i j}$.

Step 6: If $(k>N)$, then

$$
\text { Next } j
$$

else

\section{Goto Step 2}

End if

End For $j$

End For Loop

3. Main results on cyclic-12

The system cyclic-12 with level one of cascade, $l=1$, is an embedded system of size 13 which can be solved numerically by HOM4PS-2.0 [15]. The number of true witness points of this system is 144 . These 144 points came out of a system with a mixed volume equaling 983,952 . To search for witness points on dimensions two and three, we ran the code with $l=2,3$. No witness point appeared.

A typical monodromy break up of 144 witness points for this system is given as

$$
144 \rightarrow 109 \rightarrow 101 \rightarrow 86 \rightarrow 74 \rightarrow 73 \rightarrow 73 \rightarrow 72 \rightarrow 72 \rightarrow 72 \ldots
$$

Therefore, we conclude (based on this numerical decomposition) that we have 72 numerical irreducible components of second degree on dimension one. We write

$$
W=W_{1}^{2} \cup \ldots \cup W_{72}^{2} .
$$

To form linear generic matrices for each of the 72 components, we need 13 well-separated points starting from one sample point on each component. In this example, we used (19) and (20) as separation strategies with $0.01 \leqslant \alpha_{2}, \alpha_{\infty} \leqslant 0.12$ uniformly for all components. We see that all linear generic matrices are of rank three. These 72 components will be partitioned into 12 groups $C_{1}, \ldots, C_{12}$.

As a more specific example, the zero vectors of $\mathbf{A}_{L i n G}$ corresponding to $W_{1}^{2}$ are

$$
\begin{gathered}
(1,0,0,0,0,0,0,0,0,0,-\bar{\omega}, 0,0),(0,1,0,0,0,0,0,0,0,0,0,-\bar{\omega}, 0), \\
(0,0,1,0,0,0,0,0,0,0, \omega, 0,0),(0,0,0,1,0,0,0,0,0,0,0, \omega, 0), \\
(0,0,0,0,1,0,0,0,0,0,1,0,0),(0,0,0,0,0,1,0,0,0,0,0,1,0), \\
(0,0,0,0,0,0,1,0,0,0, \bar{\omega}, 0,0),(0,0,0,0,0,0,0,1,0,0,0, \bar{\omega}, 0), \\
(0,0,0,0,0,0,0,0,1,0,-\omega, 0,0),(0,0,0,0,0,0,0,0,0,1,0,-\omega, 0),
\end{gathered}
$$

where $\omega:=\frac{1}{2}-i \frac{\sqrt{3}}{2}$. In actual numerical calculation of the above vectors, we reached an approximation of the algebraic number $\frac{\sqrt{3}}{2}(\approx 0.866025403784439)$, which appeared in a related idea of tropism in [27].

These zeros correspond to the following linear equations:

$$
\begin{array}{llll}
\tilde{p}_{1}=x_{1}-\bar{\omega} x_{11}, & \tilde{p}_{2}=x_{2}-\bar{\omega} x_{12}, & \tilde{p}_{3}=x_{3}+\omega x_{11}, & \tilde{p}_{4}=x_{4}+\omega x_{12}, \\
\tilde{p}_{5}=x_{5}+x_{11}, & \tilde{p}_{6}=x_{6}+x_{12}, & \tilde{p}_{7}=x_{7}+\bar{\omega} x_{11}, & \tilde{p}_{8}=x_{8}+\bar{\omega} x_{12}, \\
\tilde{p}_{9}=x_{9}-\omega x_{11}, & \tilde{p}_{10}=x_{10}-\omega x_{12} . & &
\end{array}
$$


Based on the first two polynomials, we may express the variables $x_{3}, \ldots, x_{10}$ in terms of $x_{1}$ and $x_{2}$. We have

$$
\begin{array}{llll}
p_{1}=x_{1}-\omega x_{3}, & p_{2}=x_{2}-\omega x_{4}, & p_{3}=x_{1}+\bar{\omega} x_{5}, & p_{4}=x_{2}+\bar{\omega} x_{6}, \\
p_{5}=x_{1}+x_{7}, & p_{6}=x_{2}+x_{8}, & p_{7}=x_{1}+\omega x_{9}, & p_{8}=x_{2}+\omega x_{10}, \\
p_{9}=x_{1}-\bar{\omega} x_{11}, & p_{10}=x_{2}-\bar{\omega} x_{12} . & &
\end{array}
$$

These 10 linear polynomials will be shared among six components, which makes group $C_{1}$. The members of $C_{1}$ differ by the last (11th) polynomial, which is quadratic. Since $p_{1}, \ldots, p_{10}$ in the above set of polynomials have been expressed in terms of $x_{1}$ and $x_{2}$, we may set up the quadratic generic matrix $\mathbf{A}_{Q u a G}$ based on the monomials $x_{1}^{2}, x_{1} x_{2}, x_{2}^{2}, x_{1}, x_{2}, 1$ (keeping the order). The corresponding $\mathbf{A}_{Q u a G}$ for each component in $C_{1}$ has only one zero vector. For six members of $C_{1}$, we get the following zeros:

$$
(0,1,0,0,0, \pm 1), \quad(0,1,0,0,0, \pm \omega), \quad(0,1,0,0,0, \pm \bar{\omega}) .
$$

Therefore, we may denote the exact irreducible decomposition of cyclic-12 by

$$
V=V_{C_{1}}^{ \pm 1} \cup V_{C_{1}}^{ \pm \omega} \cup V_{C_{1}}^{ \pm \bar{\omega}} \cup \ldots \cup V_{C_{12}}^{ \pm 1} \cup V_{C_{12}}^{ \pm \omega} \cup V_{C_{12}}^{ \pm \bar{\omega}} \text {. }
$$

Below, we list all of the corresponding 72 ideals with their generators we derived. See the Appendix for polynomial expressions of some $f_{1}, \ldots, f_{5}$ in (3) in terms of generators of $\mathbf{I}_{C_{1}}^{ \pm 1}$.

$$
\begin{aligned}
& \mathbf{I}_{C_{1}}^{ \pm 1}:=\left\langle x_{1}-\omega x_{3}, x_{2}-\omega x_{4}, x_{1}+\bar{\omega} x_{5}, x_{2}+\bar{\omega} x_{6}, x_{1}+x_{7}, x_{2}+x_{8}, x_{1}+\omega x_{9},\right. \\
& \left.x_{2}+\omega x_{10}, x_{1}-\bar{\omega} x_{11}, x_{2}-\bar{\omega} x_{12}, x_{1} x_{2} \pm 1\right\rangle, \\
& \mathbf{I}_{C_{1}}^{ \pm \bar{\omega}}:=\left\langle x_{1}-\omega x_{3}, x_{2}-\omega x_{4}, x_{1}+\bar{\omega} x_{5}, x_{2}+\bar{\omega} x_{6}, x_{1}+x_{7}, x_{2}+x_{8}, x_{1}+\omega x_{9},\right. \\
& \left.x_{2}+\omega x_{10}, x_{1}-\bar{\omega} x_{11}, x_{2}-\bar{\omega} x_{12}, x_{1} x_{2} \pm \bar{\omega}\right\rangle, \\
& \mathbf{I}_{C_{1}}^{ \pm \omega}:=\left\langle x_{1}-\omega x_{3}, x_{2}-\omega x_{4}, x_{1}+\bar{\omega} x_{5}, x_{2}+\bar{\omega} x_{6}, x_{1}+x_{7}, x_{2}+x_{8}, x_{1}+\omega x_{9},\right. \\
& \left.x_{2}+\omega x_{10}, x_{1}-\bar{\omega} x_{11}, x_{2}-\bar{\omega} x_{12}, x_{1} x_{2} \pm \omega\right\rangle, \\
& \mathbf{I}_{C_{2}}^{ \pm 1}:=\left\langle x_{1}-\omega x_{3}, x_{2}+x_{4}, x_{1}-x_{5}, x_{2}+\bar{\omega} x_{6}, x_{1}+x_{7}, x_{2}+x_{8}, x_{1}+\omega x_{9},\right. \\
& \left.x_{2}-x_{10}, x_{1}+x_{11}, x_{2}-\bar{\omega} x_{12}, x_{1} x_{2} \pm 1\right\rangle, \\
& \mathbf{I}_{C_{2}}^{ \pm \bar{\omega}}:=\left\langle x_{1}-\omega x_{3}, x_{2}+x_{4}, x_{1}-x_{5}, x_{2}+\bar{\omega} x_{6}, x_{1}+x_{7}, x_{2}+x_{8}, x_{1}+\omega x_{9},\right. \\
& \left.x_{2}-x_{10}, x_{1}+x_{11}, x_{2}-\bar{\omega} x_{12}, x_{1} x_{2} \pm \bar{\omega}\right\rangle, \\
& \mathbf{I}_{C_{2}}^{ \pm \omega}:=\left\langle x_{1}-\omega x_{3}, x_{2}+x_{4}, x_{1}-x_{5}, x_{2}+\bar{\omega} x_{6}, x_{1}+x_{7}, x_{2}+x_{8}, x_{1}+\omega x_{9}\right. \text {, } \\
& \left.x_{2}-x_{10}, x_{1}+x_{11}, x_{2}-\bar{\omega} x_{12}, x_{1} x_{2} \pm \omega\right\rangle \text {, } \\
& \mathbf{I}_{C_{3}}^{ \pm 1}:=\left\langle x_{1}-\bar{\omega} x_{3}, x_{2}+x_{4}, x_{1}-x_{5}, x_{2}+\omega x_{6}, x_{1}+x_{7}, x_{2}+x_{8}, x_{1}+\bar{\omega} x_{9},\right. \\
& \left.x_{2}-x_{10}, x_{1}+x_{11}, x_{2}-\omega x_{12}, x_{1} x_{2} \pm 1\right\rangle, \\
& \mathbf{I}_{C_{3}}^{ \pm \bar{\omega}}:=\left\langle x_{1}-\bar{\omega} x_{3}, x_{2}+x_{4}, x_{1}-x_{5}, x_{2}+\omega x_{6}, x_{1}+x_{7}, x_{2}+x_{8}, x_{1}+\bar{\omega} x_{9},\right. \\
& \left.x_{2}-x_{10}, x_{1}+x_{11}, x_{2}-\omega x_{12}, x_{1} x_{2} \pm \bar{\omega}\right\rangle, \\
& \mathbf{I}_{C_{3}}^{ \pm \omega}:=\left\langle x_{1}-\bar{\omega} x_{3}, x_{2}+x_{4}, x_{1}-x_{5}, x_{2}+\omega x_{6}, x_{1}+x_{7}, x_{2}+x_{8}, x_{1}+\bar{\omega} x_{9},\right. \\
& \left.x_{2}-x_{10}, x_{1}+x_{11}, x_{2}-\omega x_{12}, x_{1} x_{2} \pm \omega\right\rangle \text {, } \\
& \mathbf{I}_{C_{4}}^{ \pm 1}:=\left\langle x_{1}-\bar{\omega} x_{3}, x_{2}-\bar{\omega} x_{4}, x_{1}+\omega x_{5}, x_{2}+\omega x_{6}, x_{1}+x_{7}, x_{2}+x_{8}, x_{1}+\bar{\omega} x_{9},\right. \\
& \left.x_{2}+\bar{\omega} x_{10}, x_{1}-\omega x_{11}, x_{2}-\omega x_{12}, x_{1} x_{2} \pm 1\right\rangle, \\
& \mathbf{I}_{C_{4}}^{ \pm \bar{\omega}}:=\left\langle x_{1}-\bar{\omega} x_{3}, x_{2}-\bar{\omega} x_{4}, x_{1}+\omega x_{5}, x_{2}+\omega x_{6}, x_{1}+x_{7}, x_{2}+x_{8}, x_{1}+\bar{\omega} x_{9},\right. \\
& \left.x_{2}+\bar{\omega} x_{10}, x_{1}-\omega x_{11}, x_{2}-\omega x_{12}, x_{1} x_{2} \pm \bar{\omega}\right\rangle, \\
& \mathbf{I}_{C_{4}}^{ \pm \omega}:=\left\langle x_{1}-\bar{\omega} x_{3}, x_{2}-\bar{\omega} x_{4}, x_{1}+\omega x_{5}, x_{2}+\omega x_{6}, x_{1}+x_{7}, x_{2}+x_{8}, x_{1}+\bar{\omega} x_{9},\right. \\
& \left.x_{2}+\bar{\omega} x_{10}, x_{1}-\omega x_{11}, x_{2}-\omega x_{12}, x_{1} x_{2} \pm \omega\right\rangle,
\end{aligned}
$$




$$
\begin{aligned}
& \mathbf{I}_{C_{5}}^{ \pm 1}:=\left\langle x_{1}+x_{3}, x_{2}-\bar{\omega} x_{4}, x_{1}+\bar{\omega} x_{5}, x_{2}+\bar{\omega} x_{6}, x_{1}+x_{7}, x_{2}+x_{8}, x_{1}-x_{9},\right. \\
& \left.x_{2}+\bar{\omega} x_{10}, x_{1}-\bar{\omega} x_{11}, x_{2}-\bar{\omega} x_{12}, x_{1} x_{2} \pm 1\right\rangle \text {, } \\
& \mathbf{I}_{C_{5}}^{ \pm \bar{\omega}}:=\left\langle x_{1}+x_{3}, x_{2}-\bar{\omega} x_{4}, x_{1}+\bar{\omega} x_{5}, x_{2}+\bar{\omega} x_{6}, x_{1}+x_{7}, x_{2}+x_{8}, x_{1}-x_{9},\right. \\
& \left.x_{2}+\bar{\omega} x_{10}, x_{1}-\bar{\omega} x_{11}, x_{2}-\bar{\omega} x_{12}, x_{1} x_{2} \pm \bar{\omega}\right\rangle, \\
& \mathbf{I}_{C_{5}}^{ \pm \omega}:=\left\langle x_{1}+x_{3}, x_{2}-\bar{\omega} x_{4}, x_{1}+\bar{\omega} x_{5}, x_{2}+\bar{\omega} x_{6}, x_{1}+x_{7}, x_{2}+x_{8}, x_{1}-x_{9},\right. \\
& \left.x_{2}+\bar{\omega} x_{10}, x_{1}-\bar{\omega} x_{11}, x_{2}-\bar{\omega} x_{12}, x_{1} x_{2} \pm \omega\right\rangle \text {, } \\
& \mathbf{I}_{C_{6}}^{ \pm 1}:=\left\langle x_{1}-\bar{\omega} x_{3}, x_{2}+x_{4}, x_{1}+\omega x_{5}, x_{2}-x_{6}, x_{1}+x_{7}, x_{2}+x_{8}, x_{1}+\bar{\omega} x_{9},\right. \\
& \left.x_{2}-x_{10}, x_{1}-\omega x_{11}, x_{2}+x_{12}, x_{1} x_{2} \pm 1\right\rangle \text {, } \\
& \mathbf{I}_{C_{6}}^{ \pm \bar{\omega}}:=\left\langle x_{1}-\bar{\omega} x_{3}, x_{2}+x_{4}, x_{1}+\omega x_{5}, x_{2}-x_{6}, x_{1}+x_{7}, x_{2}+x_{8}, x_{1}+\bar{\omega} x_{9},\right. \\
& \left.x_{2}-x_{10}, x_{1}-\omega x_{11}, x_{2}+x_{12}, x_{1} x_{2} \pm \bar{\omega}\right\rangle, \\
& \mathbf{I}_{C_{6}}^{ \pm \omega}:=\left\langle x_{1}-\bar{\omega} x_{3}, x_{2}+x_{4}, x_{1}+\omega x_{5}, x_{2}-x_{6}, x_{1}+x_{7}, x_{2}+x_{8}, x_{1}+\bar{\omega} x_{9},\right. \\
& \left.x_{2}-x_{10}, x_{1}-\omega x_{11}, x_{2}+x_{12}, x_{1} x_{2} \pm \omega\right\rangle \text {, } \\
& \mathbf{I}_{C_{7}}^{ \pm 1}:=\left\langle x_{1}-\omega x_{3}, x_{2}+x_{4}, x_{1}+\bar{\omega} x_{5}, x_{2}-x_{6}, x_{1}+x_{7}, x_{2}+x_{8}, x_{1}+\omega x_{9},\right. \\
& \left.x_{2}-x_{10}, x_{1}-\bar{\omega} x_{11}, x_{2}+x_{12}, x_{1} x_{2} \pm 1\right\rangle, \\
& \mathbf{I}_{C_{7}}^{ \pm \bar{\omega}}:=\left\langle x_{1}-\omega x_{3}, x_{2}+x_{4}, x_{1}+\bar{\omega} x_{5}, x_{2}-x_{6}, x_{1}+x_{7}, x_{2}+x_{8}, x_{1}+\omega x_{9},\right. \\
& \left.x_{2}-x_{10}, x_{1}-\bar{\omega} x_{11}, x_{2}+x_{12}, x_{1} x_{2} \pm \bar{\omega}\right\rangle, \\
& \mathbf{I}_{C_{7}}^{ \pm \omega}:=\left\langle x_{1}-\omega x_{3}, x_{2}+x_{4}, x_{1}+\bar{\omega} x_{5}, x_{2}-x_{6}, x_{1}+x_{7}, x_{2}+x_{8}, x_{1}+\omega x_{9},\right. \\
& \left.x_{2}-x_{10}, x_{1}-\bar{\omega} x_{11}, x_{2}+x_{12}, x_{1} x_{2} \pm \omega\right\rangle \text {, } \\
& \mathbf{I}_{C_{8}}^{ \pm 1}:=\left\langle x_{1}-\bar{\omega} x_{3}, x_{2}-\bar{\omega} x_{4}, x_{1}+\bar{\omega} x_{5}, x_{2}-x_{6}, x_{1}+x_{7}, x_{2}+x_{8}, x_{1}+\bar{\omega} x_{9},\right. \\
& \left.x_{2}+\bar{\omega} x_{10}, x_{1}-\bar{\omega} x_{11}, x_{2}+x_{12}, x_{1} x_{2} \pm 1\right\rangle, \\
& \mathbf{I}_{C_{8}}^{ \pm \bar{\omega}}:=\left\langle x_{1}-\bar{\omega} x_{3}, x_{2}-\bar{\omega} x_{4}, x_{1}+\bar{\omega} x_{5}, x_{2}-x_{6}, x_{1}+x_{7}, x_{2}+x_{8}, x_{1}+\bar{\omega} x_{9},\right. \\
& \left.x_{2}+\bar{\omega} x_{10}, x_{1}-\bar{\omega} x_{11}, x_{2}+x_{12}, x_{1} x_{2} \pm \bar{\omega}\right\rangle, \\
& \mathbf{I}_{C_{8}}^{ \pm \omega}:=\left\langle x_{1}-\bar{\omega} x_{3}, x_{2}-\bar{\omega} x_{4}, x_{1}+\bar{\omega} x_{5}, x_{2}-x_{6}, x_{1}+x_{7}, x_{2}+x_{8}, x_{1}+\bar{\omega} x_{9},\right. \\
& \left.x_{2}+\bar{\omega} x_{10}, x_{1}-\bar{\omega} x_{11}, x_{2}+x_{12}, x_{1} x_{2} \pm \omega\right\rangle, \\
& \mathbf{I}_{C_{9}}^{ \pm 1}:=\left\langle x_{1}+x_{3}, x_{2}-\omega x_{4}, x_{1}-x_{5}, x_{2}+\bar{\omega} x_{6}, x_{1}+x_{7}, x_{2}+x_{8}, x_{1}-x_{9},\right. \\
& \left.x_{2}+\omega x_{10}, x_{1}+x_{11}, x_{2}-\bar{\omega} x_{12}, x_{1} x_{2} \pm 1\right\rangle \text {, } \\
& \mathbf{I}_{C_{9}}^{ \pm \bar{\omega}}:=\left\langle x_{1}+x_{3}, x_{2}-\omega x_{4}, x_{1}-x_{5}, x_{2}+\bar{\omega} x_{6}, x_{1}+x_{7}, x_{2}+x_{8}, x_{1}-x_{9},\right. \\
& \left.x_{2}+\omega x_{10}, x_{1}+x_{11}, x_{2}-\bar{\omega} x_{12}, x_{1} x_{2} \pm \bar{\omega}\right\rangle, \\
& \mathbf{I}_{C_{9}}^{ \pm \omega}:=\left\langle x_{1}+x_{3}, x_{2}-\omega x_{4}, x_{1}-x_{5}, x_{2}+\bar{\omega} x_{6}, x_{1}+x_{7}, x_{2}+x_{8}, x_{1}-x_{9},\right. \\
& \left.x_{2}+\omega x_{10}, x_{1}+x_{11}, x_{2}-\bar{\omega} x_{12}, x_{1} x_{2} \pm \omega\right\rangle \text {, } \\
& \mathbf{I}_{C_{10}}^{ \pm 1}:=\left\langle x_{1}+x_{3}, x_{2}-\bar{\omega} x_{4}, x_{1}-x_{5}, x_{2}+\omega x_{6}, x_{1}+x_{7}, x_{2}+x_{8}, x_{1}-x_{9},\right. \\
& \left.x_{2}+\bar{\omega} x_{10}, x_{1}+x_{11}, x_{2}-\omega x_{12}, x_{1} x_{2} \pm 1\right\rangle \text {, } \\
& \mathbf{I}_{C_{10}}^{ \pm \bar{\omega}}:=\left\langle x_{1}+x_{3}, x_{2}-\bar{\omega} x_{4}, x_{1}-x_{5}, x_{2}+\omega x_{6}, x_{1}+x_{7}, x_{2}+x_{8}, x_{1}-x_{9}\right. \text {, } \\
& \left.x_{2}+\bar{\omega} x_{10}, x_{1}+x_{11}, x_{2}-\omega x_{12}, x_{1} x_{2} \pm \bar{\omega}\right\rangle, \\
& \mathbf{I}_{C_{10}}^{ \pm \omega}:=\left\langle x_{1}+x_{3}, x_{2}-\bar{\omega} x_{4}, x_{1}-x_{5}, x_{2}+\omega x_{6}, x_{1}+x_{7}, x_{2}+x_{8}, x_{1}-x_{9}\right. \text {, } \\
& \left.x_{2}+\bar{\omega} x_{10}, x_{1}+x_{11}, x_{2}-\omega x_{12}, x_{1} x_{2} \pm \omega\right\rangle \text {, } \\
& \mathbf{I}_{C_{11}}^{ \pm 1}:=\left\langle x_{1}-\omega x_{3}, x_{2}-\omega x_{4}, x_{1}+\omega x_{5}, x_{2}-x_{6}, x_{1}+x_{7}, x_{2}+x_{8}, x_{1}+\omega x_{9},\right. \\
& \left.x_{2}+\omega x_{10}, x_{1}-\omega x_{11}, x_{2}+x_{12}, x_{1} x_{2} \pm 1\right\rangle \text {, }
\end{aligned}
$$




$$
\begin{aligned}
\mathbf{I}_{C_{11}}^{ \pm \bar{\omega}}:= & \left\langle x_{1}-\omega x_{3}, x_{2}-\omega x_{4}, x_{1}+\omega x_{5}, x_{2}-x_{6}, x_{1}+x_{7}, x_{2}+x_{8}, x_{1}+\omega x_{9},\right. \\
& \left.x_{2}+\omega x_{10}, x_{1}-\omega x_{11}, x_{2}+x_{12}, x_{1} x_{2} \pm \bar{\omega}\right\rangle \\
\mathbf{I}_{C_{11}}^{ \pm \omega}:= & \left\langle x_{1}-\omega x_{3}, x_{2}-\omega x_{4}, x_{1}+\omega x_{5}, x_{2}-x_{6}, x_{1}+x_{7}, x_{2}+x_{8}, x_{1}+\omega x_{9},\right. \\
& \left.x_{2}+\omega x_{10}, x_{1}-\omega x_{11}, x_{2}+x_{12}, x_{1} x_{2} \pm \omega\right\rangle \\
\mathbf{I}_{C_{12}}^{ \pm 1}:= & \left\langle x_{1}+x_{3}, x_{2}-\omega x_{4}, x_{1}+\omega x_{5}, x_{2}+\omega x_{6}, x_{1}+x_{7}, x_{2}+x_{8}, x_{1}-x_{9},\right. \\
& \left.x_{2}+\omega x_{10}, x_{1}-\omega x_{11}, x_{2}-\omega x_{12}, x_{1} x_{2} \pm 1\right\rangle \\
\mathbf{I}_{C_{12}}^{ \pm \bar{\omega}}:= & \left\langle x_{1}+x_{3}, x_{2}-\omega x_{4}, x_{1}+\omega x_{5}, x_{2}+\omega x_{6}, x_{1}+x_{7}, x_{2}+x_{8}, x_{1}-x_{9},\right. \\
& \left.x_{2}+\omega x_{10}, x_{1}-\omega x_{11}, x_{2}-\omega x_{12}, x_{1} x_{2} \pm \bar{\omega}\right\rangle \\
\mathbf{I}_{C_{12}}^{ \pm \omega}:= & \left\langle x_{1}+x_{3}, x_{2}-\omega x_{4}, x_{1}+\omega x_{5}, x_{2}+\omega x_{6}, x_{1}+x_{7}, x_{2}+x_{8}, x_{1}-x_{9},\right. \\
& \left.x_{2}+\omega x_{10}, x_{1}-\omega x_{11}, x_{2}-\omega x_{12}, x_{1} x_{2} \pm \omega\right\rangle .
\end{aligned}
$$

TheOREm 3.1. All of the above 72 ideals of cyclic-12 are prime and of codimension 11.

Proof. We take one typical ideal. Let

$$
\begin{aligned}
\mathbf{I}_{C_{1}}^{\omega}:= & \left\langle x_{1}-\omega x_{3}, x_{2}-\omega x_{4}, x_{1}+\bar{\omega} x_{5}, x_{2}+\bar{\omega} x_{6}, x_{1}+x_{7}, x_{2}+x_{8}, x_{1}+\omega x_{9},\right. \\
& \left.x_{2}+\omega x_{10}, x_{1}-\bar{\omega} x_{11}, x_{2}-\bar{\omega} x_{12}, x_{1} x_{2}+\omega\right\rangle=\left\langle\xi_{1}, \ldots, \xi_{10}, \eta\right\rangle,
\end{aligned}
$$

where

$$
\begin{aligned}
& \xi_{1}:=x_{1}-\omega x_{3}, \xi_{2}:=x_{2}-\omega x_{4}, \xi_{3}:=x_{1}+\bar{\omega} x_{5}, \xi_{4}:=x_{2}+\bar{\omega} x_{6}, \xi_{5}:=x_{1}+x_{7}, \xi_{6}:=x_{2}+x_{8}, \\
& \xi_{7}:=x_{1}+\omega x_{9}, \xi_{8}:=x_{2}+\omega x_{10}, \xi_{9}:=x_{1}-\bar{\omega} x_{11}, \xi_{10}:=x_{2}-\bar{\omega} x_{12}, \eta:=x_{1} x_{2}+\omega .
\end{aligned}
$$

We define

$$
\begin{aligned}
\hat{\xi}_{1} & :=\xi_{9}=x_{1}-\bar{\omega} x_{11}, \\
\hat{\xi}_{2} & :=\xi_{10}=x_{2}-\bar{\omega} x_{12}, \\
\hat{\xi}_{3} & :=\left(\xi_{1}-\xi_{9}\right) /(-\omega)=x_{3}+\omega x_{11}, \\
\hat{\xi}_{4} & :=\left(\xi_{2}-\xi_{10}\right) /(-\omega)=x_{4}+\omega x_{12}, \\
\hat{\xi}_{5} & :=\left(\xi_{3}-\xi_{9}\right) / \bar{\omega}=x_{5}+x_{11}, \\
\hat{\xi}_{6} & :=\left(\xi_{4}-\xi_{10}\right) / \bar{\omega}=x_{6}+x_{12}, \\
\hat{\xi}_{7} & :=\xi_{5}-\xi_{9}=x_{7}+\bar{\omega} x_{11} \\
\hat{\xi}_{8} & :=\xi_{6}-\xi_{10}=x_{8}+\bar{\omega} x_{12}, \\
\hat{\xi}_{9} & :=\left(\xi_{7}-\xi_{9}\right) / \omega=x_{9}-\omega x_{11}, \\
\hat{\xi}_{10} & :=\left(\xi_{8}-\xi_{10}\right) / \omega=x_{10}-\omega x_{12}, \\
\hat{\eta} & :=\left(\eta-x_{2} \xi_{9}-x_{1} \xi_{10}+\xi_{9} \xi_{10}\right) /(-\omega)=x_{11} x_{12}-1 .
\end{aligned}
$$

Let $f \in \mathbf{I}_{\mathcal{C}_{1}}^{\omega}$ be defined as $f:=g_{1} \xi_{1}+\ldots+g_{10} \xi_{10}+g \eta$, where $g_{1}, \ldots, g_{10}, g \in \mathbb{C}\left[x_{1}, \ldots, x_{12}\right]$. Then, by adding and subtracting appropriate polynomial combinations of $\xi_{9}$ and $\xi_{10}$ to each term, we may consider $f$ as $f:=\hat{g}_{1} \hat{\xi}_{1}+\ldots+\hat{g}_{10} \hat{\xi}_{10}+\hat{g} \hat{\eta}$, where $\hat{g}_{1}, \ldots, \hat{g}_{10}, \hat{g} \in \mathbb{C}\left[x_{1}, \ldots, x_{12}\right]$. We show that $\mathbf{I}_{\mathcal{C}_{1}}^{\omega}$ can be written as

$$
\begin{aligned}
\mathbf{I}:= & \left\langle x_{1}+\beta_{1} x_{11}, x_{2}+\beta_{2} x_{12}, x_{3}+\beta_{3} x_{11}, x_{4}+\beta_{4} x_{12}, x_{5}+\beta_{5} x_{11}, x_{6}+\beta_{6} x_{12},\right. \\
& \left.x_{7}+\beta_{7} x_{11}, x_{8}+\beta_{8} x_{12}, x_{9}+\beta_{9} x_{11}, x_{10}+\beta_{10} x_{12}, x_{11} x_{12}+K\right\rangle
\end{aligned}
$$

for suitable non-zero constants $\beta_{1}, \ldots, \beta_{10}, K$ in $\mathbb{C}$.

Straightforward calculations with the Buchberger algorithm [11] show that the ideal of the form (25) is a minimal Groebner basis with respect to lexicographic ordering $x_{1}>\ldots>x_{12}$. For $f, g \in \mathbb{C}\left[x_{1}, \ldots, x_{12}\right]$, let $f g \in \mathbf{I}$. We need to show whether $f \in \mathbf{I}$ or $g \in \mathbf{I}$. Since (25) is a minimal Groebner basis, then, on division of $f$ and $g$ by $\mathbf{I}$, we have $f=f_{\xi}+F$ and $g=g_{\xi}+G$, where $f_{\xi}$ and $g_{\xi}$ are combinations of the $\hat{\xi}$, and $F$ and $G$ are unique remainders on division 
of $f$ and $g$ by $\mathbf{I}$, and are denoted by $\bar{f}^{\mathbf{I}}$ and $\bar{g}^{\mathbf{I}}$, respectively. Note that $\overline{f g}^{\mathbf{I}}=0$ means $\overline{F G}^{\mathbf{I}}=0$. Suppose $F \neq 0$; we will show $G=0$.

Since $\overline{F G}^{\mathbf{I}}=0$, no monomial in $F$ and $G$ is divisible by leading monomials of $\hat{\xi}_{1}, \ldots, \hat{\xi}_{10}, \hat{\eta}$ $\left(x_{1}, \ldots, x_{10}, x_{11} x_{12}\right.$, respectively). This means that the forms of $F$ and $G$ are as follows:

$$
\begin{aligned}
& F:=\Sigma_{11}+\Sigma_{12}+a_{0}, \\
& G:=\Sigma_{11}^{\prime}+\Sigma_{12}^{\prime}+b_{0},
\end{aligned}
$$

where, for positive integers $m, n, k, l$, all $\geqslant 1$ and complex constants $a_{i}, a_{j}^{\prime}, b_{t}, b_{s}^{\prime}$ (to be determined), we let

$$
\begin{aligned}
& \Sigma_{11}:=a_{1} x_{11}+\ldots+a_{n} x_{11}^{n}, \\
& \Sigma_{12}:=b_{1} x_{12}+\ldots+b_{m} x_{12}^{m}, \\
& \Sigma_{11}^{\prime}:=a_{1}^{\prime} x_{11}+\ldots+a_{k}^{\prime} x_{11}^{k}, \\
& \Sigma_{12}^{\prime}:=b_{1}^{\prime} x_{12}+\ldots+b_{l}^{\prime} x_{12}^{l} .
\end{aligned}
$$

To prove $G=0$, we need to show $a_{1}^{\prime}=\ldots=a_{k}^{\prime}=b_{1}^{\prime}=\ldots=b_{l}^{\prime}=b_{0}=0$. Since $F G$ does not have any term involving $x_{1}, \ldots, x_{10}$, we have $\overline{F G}^{\mathbf{I}}=\overline{F G}^{\left\{x_{11} x_{12}+K\right\}}$. Therefore,

$$
\begin{aligned}
\overline{F G}^{\mathbf{I}}= & \Sigma_{11} \Sigma_{11}^{\prime}+\overline{\Sigma_{11}^{\prime} \Sigma_{12}}\left\{x_{11} x_{12}+K\right\}+\overline{\Sigma_{11} \Sigma_{12}^{\prime}}\left\{x_{11} x_{12}+K\right\} \\
& +\Sigma_{12} \Sigma_{12}^{\prime}+a_{0} \Sigma_{11}^{\prime}+b_{0} \Sigma_{11}+a_{0} \Sigma_{12}^{\prime}+b_{0} \Sigma_{12}+a_{0} b_{0} .
\end{aligned}
$$

Assume $l>m$ and $k>n$. The other cases are similar.

We consider two different sorted expansions of $\overline{F G}^{\mathbf{I}}$ once with decreasing powers of $x_{11}$ and another time with decreasing powers of $x_{12}$. Note that the powers of (say) $x_{11}$ are among the terms in the expansions $\Sigma_{11} \Sigma_{11}^{\prime}, \overline{\Sigma_{11}^{\prime} \Sigma_{12}}\left\{x_{11} x_{12}+K\right\}$ and $\overline{\Sigma_{11} \Sigma_{12}^{\prime}}\left\{x_{11} x_{12}+K\right\}$.

The coefficients of $x_{11}^{n+k}, x_{11}^{n+k-1}, \ldots, x_{11}^{k}$ in the first expansion (decreasing powers of $x_{11}$ ) respectively are $a_{n} a_{k}^{\prime}, a_{n} a_{k-1}^{\prime}+a_{n-1} a_{k}^{\prime}, \ldots, a_{n} a_{k-n}^{\prime}+\ldots+a_{1} a_{k}^{\prime}+a_{0} a_{k}^{\prime}$. Setting them equal to zero one at a time gives rise to the conclusion $a_{k}^{\prime}=a_{k-1}^{\prime}=\ldots=a_{k-n}^{\prime}=0$. Similarly, by setting the coefficients of $x_{12}^{l+m}, x_{12}^{l+m-1}, \ldots, x_{12}^{l}$ equal to zero we get $b_{l}^{\prime}=b_{l-1}^{\prime}=\ldots=b_{l-m}^{\prime}=0$.

Now we consider the coefficients of $x_{11}^{k-1}, \ldots, x_{11}$ one at a time and from left to right. The coefficients of $x_{11}^{k-1}$ contain the terms involving $a_{k}^{\prime}, a_{k-1}^{\prime}, \ldots, a_{k-n}^{\prime}$, which are all zero, and a term involving $a_{k}^{\prime} b_{1}$ and $a_{n} a_{k-n-1}^{\prime}$, which implies $a_{k-n-1}=0$. Continuing in this way, we may conclude that all $a_{j}^{\prime}$ (including $b_{0}$ ) are zero. On the other hand, for coefficients of the powers of $x_{12}$, we may conclude that all $b_{s}^{\prime}$ are zero. This would end the proof of the primality part of the argument.

Now consider the zero set of this ideal:

$$
\begin{aligned}
Z\left(\mathbf{I}_{\mathcal{C}_{1}}^{\omega}\right) & :=\left\{\left(x_{1}, \ldots, x_{12}\right) \in \mathbb{C}^{12}: f\left(x_{1}, \ldots, x_{12}\right)=0, \forall f \in \mathbf{I}_{\mathcal{C}_{1}}^{\omega}\right\} \\
& :=\left\{\left(x_{1}, \ldots, x_{12}\right) \in \mathbb{C}^{12}: \hat{\xi}_{1}=0, \ldots, \hat{\xi}_{10}=0, \hat{\eta}=0\right\} \\
& :=V\left(\hat{\xi}_{1}, \ldots, \hat{\xi}_{10}, \hat{\eta}\right) .
\end{aligned}
$$

From $\hat{\eta}=0$, we can set $x_{12}=\omega / x_{11}$ and all other defining polynomials in $\hat{\xi}_{1}, \ldots, \hat{\xi}_{10}$ can be written in terms of one parameter $x_{11}$. Therefore, $V\left(\hat{\xi}_{1}, \ldots, \hat{\xi}_{10}, \hat{\eta}\right)$, and in turn $\mathbf{I}_{C_{1}}^{\omega}$, are of dimension one.

\section{Software and numerical data}

The computer codes to produce the exact form of the above ideals consist of many parts and are all written in FORTRAN90. Starting from a set of witness points at a given level of cascade, we wrote a code for monodromy grouping according to overdetermined homotopy (12). 
This part is implemented in our algorithm of numerical irreducible decomposition. Another part of the code is sampling and equation finder, which cover Algorithm 1.

In Algorithm 2, we let the user choose the constants $\alpha_{2}, \alpha_{\infty}$ arbitrarily. However, these values may differ from linear to quadratic generators. For the linear part of our example, we take $\alpha_{2}=\alpha_{\infty}=0.05$. The actual output of the code with satisfactory precision follows:

$$
\begin{aligned}
& a_{1}=(1.0000000000000,0.0000000000000), \\
& a_{2}=(1.0000000000000,0.0000000000000), \\
& a_{3}=(1.0000000000000,0.0000000000000), \\
& a_{4}=(1.0000000000000,0.0000000000000), \\
& a_{5}=(1.0000000000000,0.0000000000000), \\
& a_{6}=(1.0000000000000,0.0000000000000), \\
& a_{7}=(1.0000000000000,0.0000000000000), \\
& a_{8}=(1.0000000000000,0.0000000000000), \\
& a_{9}=(1.0000000000000,0.000000000000), \\
& a_{10}=(1.0000000000000,0.0000000000000),
\end{aligned}
$$

$$
\begin{aligned}
& a_{11}=(-0.5000000000000,-0.8660254037844), \\
& a_{12}=(-0.5000000000000,-0.8660254037844), \\
& a_{11}=(0.5000000000000,-0.8660254037844), \\
& a_{12}=(0.5000000000000,-0.8660254037844), \\
& a_{11}=(1.0000000000000,0.0000000000000), \\
& a_{12}=(1.0000000000000,0.0000000000000), \\
& a_{11}=(0.5000000000000,0.8660254037844), \\
& a_{12}=(0.5000000000000,0.8660254037844), \\
& a_{11}=(-0.5000000000000,0.8660254037844), \\
& a_{12}=(-0.5000000000000,0.8660254037844) .
\end{aligned}
$$

The above set of data represents (22). But, to obtain the corresponding zeros in (23), we need to increase $\alpha_{2}, \alpha_{\infty}$ to 0.1 . All modules of the software are at the research level. Executable files are available upon request.

\section{Conclusions and further research}

The algorithm presented in this paper is applicable to other polynomial systems with higherdimensional solution sets. However, more research should be done to make the algorithm have a wide range of applicability.

Beyond the above comment, we may well consider some options to calculate the exact form of real components inside a complex component. This issue was numerically solved for plane curves in [16]. Another type of symbolic-numerical algorithm was introduced in [4]. The basic needs for higher precision of data, in our case higher precision in calculation of algebraic numbers like $\omega$, convince people to put effort into developing software with such capabilities $[\mathbf{3}, \mathbf{6}]$. The same questions of exactness may well be posed for intersection of components as in numerical versions $[\mathbf{2 2}, \mathbf{2 4}]$ and also for calculation of secant and join as in [5]. Current research of the author focuses on exact factorization of large-scale multivariable complex polynomials; see [23].

Acknowledgements. The result of this paper will be appearing extensively in the author's $\mathrm{PhD}$ thesis. The author is grateful to his supervisor Professor T. Y. Li for his constant advice and help during the course of this research.

\section{Appendix}

This appendix contains polynomial expressions of the first five polynomials in the cyclic- 12 system in terms of the generators of $\mathbf{I}_{C_{1}}^{ \pm 1}$. Further expressions for this ideal and the others are available upon request.

$$
\begin{aligned}
\xi_{1}:= & x_{1}-\omega x_{3}, \xi_{2}:=x_{2}-\omega x_{4}, \xi_{3}:=x_{1}+\bar{\omega} x_{5}, \xi_{4}:=x_{2}+\bar{\omega} x_{6}, \xi_{5}:=x_{1}+x_{7}, \\
\xi_{6}:= & x_{2}+x_{8}, \xi_{7}:=x_{1}+\omega x_{9}, \xi_{8}:=x_{2}+\omega x_{10}, \xi_{9}:=x_{1}-\bar{\omega} x_{11}, \\
\xi_{10}:= & x_{2}-\bar{\omega} x_{12}, \eta:=x_{1} x_{2} \pm 1 \\
f_{1}:= & (-\bar{\omega}) \xi_{1}+(-\bar{\omega}) \xi_{2}+\omega \xi_{3}+\omega \xi_{4}+\xi_{5}+\xi_{6}+\bar{\omega} \xi_{7}+\bar{\omega} \xi_{8}-\omega \xi_{9}-\omega \xi_{10}, \\
f_{2}:= & \left(-\bar{\omega} x_{2}-\bar{\omega} x_{4}\right) \xi_{1}+\left(\omega x_{1}-\bar{\omega} x_{5}\right) \xi_{2}+\left(x_{2}+\omega x_{6}\right) \xi_{3}+\left(\bar{\omega} x_{1}+\omega x_{7}\right) \xi_{4}+\left(x_{8}-\omega x_{2}\right) \xi_{5} \\
& +\left(x_{9}-x_{1}\right) \xi_{6}+\left(-\bar{\omega} x_{2}+\bar{\omega} x_{10}\right) \xi_{7}+\left(\omega x_{1}+\bar{\omega} x_{11}\right) \xi_{8}+\left(x_{2}-\omega x_{12}\right) \xi_{9}+i \sqrt{3} \xi_{10},
\end{aligned}
$$




$$
\begin{aligned}
& f_{3}:=\left(-\bar{\omega} x_{2} x_{4}-\bar{\omega} x_{1} x_{2}-\bar{\omega} x_{4} x_{5}\right) \xi_{1}+\left(\omega x_{1} x_{5}+\omega x_{1} x_{2}-\bar{\omega} x_{5} x_{6}\right) \xi_{2} \\
& +\left(\bar{\omega} x_{1} x_{2}+\omega x_{6} x_{7}+x_{2} x_{6}\right) \xi_{3}+\left(-\omega x_{1} x_{2}+\omega x_{7} x_{8}+\bar{\omega} x_{1} x_{7}\right) \xi_{4} \\
& +\left(-\bar{\omega} x_{1} x_{2}-\omega x_{2} x_{8}+x_{8} x_{9}\right) \xi_{5}+\left(\omega x_{1} x_{2}+x_{9} x_{10}-x_{1} x_{9}\right) \xi_{6} \\
& +\left(\bar{\omega} x_{1} x_{2}-\bar{\omega} x_{2} x_{10}+\bar{\omega} x_{10} x_{11}\right) \xi_{7}+\left(-\omega x_{1} x_{2}+\omega x_{1} x_{11}+\bar{\omega} x_{11} x_{12}\right) \xi_{8} \\
& +\left(-\bar{\omega} x_{1} x_{2}-\omega x_{1} x_{12}+x_{2} x_{12}\right) \xi_{9}+\bar{\omega} x_{1}^{2} \xi_{10}, \\
& f_{4}:=\left(-\bar{\omega} x_{4} x_{5} x_{6}-\bar{\omega} x_{1} x_{2} x_{12}-\bar{\omega} x_{2} x_{4} x_{5}-\bar{\omega} x_{1} x_{2} x_{4}\right) \xi_{1} \\
& +\left(\omega x_{1}^{2} x_{2}+\omega x_{1} x_{2} x_{5}-\bar{\omega} x_{5} x_{6} x_{7}+\omega x_{1} x_{5} x_{6}\right) \xi_{2} \\
& +\left(\bar{\omega} x_{1} x_{2}^{2}+\bar{\omega} x_{1} x_{2} x_{6}+\omega x_{6} x_{7} x_{8}+x_{2} x_{6} x_{7}\right) \xi_{3} \\
& +\left(-\omega x_{1} x_{2} x_{7}+\bar{\omega} x_{1} x_{7} x_{8}+\omega x_{7} x_{8} x_{9}-x_{1}^{2} x_{2}\right) \xi_{4} \\
& +\left(x_{8} x_{9} x_{10}-\omega x_{2} x_{8} x_{9}-\bar{\omega} x_{1} x_{2} x_{8}+\omega x_{1}^{2} x_{2}\right) \xi_{5} \\
& +\left(\bar{\omega} x_{1}^{2} x_{2}+\omega x_{1} x_{2} x_{9}-x_{1} x_{9} x_{10}+x_{9} x_{10} x_{11}\right) \xi_{6} \\
& +\left(\bar{\omega} x_{1} x_{2} x_{10}+\bar{\omega} x_{10} x_{11} x_{12}-\bar{\omega} x_{2} x_{10} x_{11}-x_{1} x_{2}^{2}\right) \xi_{7} \\
& +\left(-\omega x_{1} x_{2} x_{11}+x_{1} x_{11} x_{12}+\omega x_{1}^{2} x_{2}\right) \xi_{8}+\bar{\omega} x_{1} x_{2}^{2} \xi_{9}-x_{1}^{2} x_{2} \xi_{10}, \\
& f_{5}:=\left(-\bar{\omega} x_{1} x_{2} x_{4} x_{12}-\bar{\omega} x_{2} x_{4} x_{5} x_{6}-\bar{\omega} x_{1} x_{2} x_{4} x_{5}-\bar{\omega} x_{1} x_{2} x_{11} x_{12}-\bar{\omega} x_{4} x_{5} x_{6} x_{7}\right) \xi_{1} \\
& +\left(-\bar{\omega} x_{5} x_{6} x_{7} x_{8}+\omega x_{1}^{2} x_{2} x_{12}+\omega x_{1}^{2} x_{2} x_{5}+\omega x_{1} x_{2} x_{5} x_{6}+\omega x_{1} x_{5} x_{6} x_{7}\right) \xi_{2} \\
& +\left(\omega x_{6} x_{7} x_{8} x_{9}+x_{2} x_{6} x_{7} x_{8}+\bar{\omega} x_{1}^{2} x_{2}^{2}+\bar{\omega} x_{1} x_{2} x_{6} x_{7}+\bar{\omega} x_{1} x_{2}^{2} x_{6}\right) \xi_{3} \\
& +\left(-\omega x_{1} x_{2} x_{7} x_{8}+\omega x_{7} x_{8} x_{9} x_{10}+\bar{\omega} x_{1} x_{7} x_{8} x_{9}-x_{1}^{2} x_{2}^{2}-x_{1}^{2} x_{2} x_{7}\right) \xi_{4} \\
& +\left(-\bar{\omega} x_{1} x_{2} x_{8} x_{9}-\omega x_{2} x_{8} x_{9} x_{10}+\omega x_{1} x_{2}^{2} x_{8}+x_{8} x_{9} x_{10} x_{11}+x_{1}^{2} x_{2}^{2}\right) \xi_{5} \\
& +\left(\omega x_{1} x_{2} x_{9} x_{10}+\bar{\omega} x_{1}^{2} x_{2} x_{9}-\omega x_{1}^{2} x_{2}^{2}-x_{1} x_{9} x_{10} x_{11}+x_{9} x_{10} x_{11} x_{12}\right) \xi_{6} \\
& +\left(\bar{\omega} x_{1} x_{10} x_{11} x_{12}-\bar{\omega} x_{2} x_{10} x_{11} x_{12}+\bar{\omega} x_{1} x_{2} x_{10} x_{11}+\omega x_{1}^{2} x_{2}^{2}-x_{1} x_{2}^{2} x_{10}\right) \xi_{7} \\
& +\left(\omega x_{1}^{2} x_{2} x_{11}+\omega x_{1}^{2} x_{11} x_{12}+\bar{\omega} x_{1}^{2} x_{2}^{2}+i \sqrt{3} x_{1} x_{2} x_{11} x_{12}\right) \xi_{8} \\
& +\left(-\bar{\omega} x_{1}^{2} x_{2}^{2}-\left(\frac{3}{2}+\frac{\sqrt{3} i}{2}\right) x_{1}^{2} x_{2} x_{12}+\left(\frac{3}{2}+\frac{\sqrt{3} i}{2}\right) x_{1} x_{2}^{2} x_{12}\right) \xi_{9} \\
& +\left(x_{1}^{2} x_{2}^{2}(1-i \sqrt{3})+\left(-\frac{3}{2}+\frac{\sqrt{3} i}{2}\right) x_{1}^{3} x_{2}\right) \xi_{10} .
\end{aligned}
$$

REMARK 4. An abstract of this paper has been presented at the AMS Joint Mathematical Meeting (JMM), Washington DC, 5-8 January 2009 and appeared in Abstracts of papers presented to the AMS, Vol. 30, No. 1, Issue 155, Page 216.

\section{References}

1. J. BACKelin and R. FröBerg, How to prove that there are 924 cyclic-7 roots?, Proc. ISSAC'91 (ed. S. M. Watt; ACM, New York, NY, 1991) 103-111.

2. D. J. Bates, J. D. Hauenstein, C. Peterson and A. J. Sommese, 'A numerical local dimension test for points on the solution set of a system of polynomial equations', SIAM J. Numer. Anal. 47 (2009) no. 5, 3608-3623.

3. D. J. Bates, J. D. Hauenstein, A. J. Sommese and C. W. Wampler, 'Software for numerical algebraic geometry: a paradigm and progress toward its implementation', Software for algebraic geometry, The IMA Volumes in Mathematics and its Applications 148 (Springer, New York, 2008) 1-14.

4. D. J. Bates, C. Peterson and A. J. Sommese, 'A numerical-symbolic algorithm for computing the multiplicity of a component of an algebraic set', J. Complexity 22 (2006) 475-489.

5. D. J. Bates, C. Peterson and A. J. Sommese, 'Applications of a numerical version of Terracini's lemma for secants and joins', Algorithms in algebraic geometry, The IMA Volumes in Mathematics and its Applications 146 (Springer, New York, 2008) 1-14.

6. D. J. Bates, A. J. Sommese, J. D. Hauenstein and C. W. Wampler, 'Adaptive multiprecision path tracking', SIAM J. Numer. Anal. 46 (2008) 722-746. 
7. D. Bini and B. Morrain, 'Polynomial test suite', INRIA Sophia Antipolis-Méditerranée, France, available at http://www-sop.inria.fr/saga/POL/BASE/2.multipol/cyclic.html.

8. G. BJÖRCK, 'Functions of modulus 1 on $\mathbb{Z}_{n}$ whose Fourier transforms have constant modulus, and cyclic $n$-roots', Recent advances in Fourier analysis and its applications (eds J. S. Byrnes and J. F. Byrnes; Kluwer Academic, Dordrecht, 1990) 131-140.

9. G. BJörCK and R. FröBerG, 'A faster way to count the solutions of inhomogeneous systems of algebraic equations, with applications to cyclic $n$-roots', J. Symbolic Comput. 12 (1991) 329-336.

10. G. BJÖRCK and R. FRÖBERG, 'Methods to 'divide out' certain solutions from systems of algebraic equations, applied to find all cyclic-8 roots', Analysis, algebra, and computers in mathematical research, Lecture Notes in Pure and Applied Mathematics 156 (Marcel Dekker, New York, 1992) 57-70.

11. D. Cox, J. LitTle and D. O'SheA, Ideals, varieties and algorithms: an introduction to computational algebraic geometry and commutative algebra, 2nd edn, Undergraduate Texts in Mathematics (Springer, New York, 1997).

12. J. C. FAUGÈRE, 'Finding all the solutions of cyclic-9 using Gröbner basis techniques', Computer mathematics (Matsuyama, 2001), Lecture Notes Series on Computing 9 (World Scientific, River Edge, NJ, 2001) 1-12.

13. R. Hartshorne, Algebraic geometry, Graduate Texts in Mathematics 52 (Springer, Berlin-New York, 1977).

14. Y.-C. KUo and T. Y. LI, 'Determine whether a numerical solution of a polynomial system is isolated', J. Math. Anal. Appl. 338 (2008) no. 2, 840-851.

15. T. LeE, T. Y. LI and C. TSAI, 'HOM4PS-2.0: a software package for solving polynomial systems by the polyhedral homotopy continuation method', Computing 83 (2008) no. 2, 109-133.

16. Y. Lu, D. J. Bates, A. J. Sommese and C. W. WAmpler, 'Finding all real points of a complex curve', Algebra, geometry, and their interactions, Contemporary Mathematics 448 (American Mathematical Society, Providence, RI, 2007) 183-205.

17. A. J. Sommese and J. Verschelde, 'Numerical homotopies to compute points on positive dimensional algebraic sets', J. Complexity 16 (2000) no. 3, 572-602.

18. A. J. Sommese, J. Verschelde and C. W. Wampler, 'Numerical decomposition of the solution sets of polynomial systems into irreducible components', SIAM J. Numer. Anal. 38 (2001) no. 6, 2022-2046.

19. A. J. Sommese, J. Verschelde and C. W. WAmpler, 'Numerical irreducible decomposition using projections from points on the components', Symbolic computation: solving equations in algebra, geometry, and engineering, Contemporary Mathematics 286 (American Mathematical Society, Providence, RI, 2001) $37-51$.

20. A. J. Sommese, J. Verschelde and C. W. Wampler, 'Using monodromy to decompose solution sets of polynomial systems into irreducible components', Applications of algebraic geometry to coding theory, physics and computation (Eilat, 2001), NATO Science Series II: Mathematics, Physics and Chemistry 36 (Springer, Dordrecht, 2001) 297-315.

21. A. J. Sommese, J. Verschelde and C. W. Wampler, 'Symmetric functions applied to decomposing solution sets of polynomial systems', SIAM J. Numer. Anal. 40 (2002) no. 6, 2026-2046.

22. A. J. Sommese, J. Verschelde and C. W. WAmpler, 'Homotopies for intersecting solution components of polynomial systems', SIAM J. Numer. Anal. 42 (2004) 1552-1571.

23. A. J. Sommese, J. Verschelde and C. W. Wampler, 'Numerical factorization of multivariate complex polynomials', Theoret. Comput. Sci. 315 (2004) 651-669.

24. A. J. Sommese, J. Verschelde and C. W. WAmpler, 'An intrinsic homotopy for intersecting algebraic varieties', J. Complexity 21 (2005) 593-608.

25. A. J. Sommese and C. W. WAMPLER, 'Numerical algebraic geometry', Mathematics of numerical analysis, Lectures in Applied Mathematics 32 (eds J. Renegar, M. Shub and S. Smale; American Mathematical Society, Providence, RI, 1996) 749-763.

26. A. J. Sommese and C. W. WAmpler, The numerical solution of systems of polynomials, arising in engineering and science (World Scientific, Hackensack, NJ, 2005).

27. J. Verschelde, 'Polyhedral methods in numerical algebraic geometry', Interaction of classical and algebraic geometry, Contemporary Mathematics 496 (eds D. Bates, G. Besana, S. DiRocco and C. Wampler; American Mathematical Society, Providence, RI, 2009) 243-263.

\author{
Rostam Sabeti \\ Department of Mathematics \\ Michigan State University \\ East Lansing, MI 48824-1027 \\ USA
}

sabetiro@math.msu.edu 